\title{
Zero Divisors among Digraphs
}

Heather Christina Smith

Virginia Commonwealth University

Follow this and additional works at: https://scholarscompass.vcu.edu/etd

Part of the Physical Sciences and Mathematics Commons

(c) The Author

\section{Downloaded from}

https://scholarscompass.vcu.edu/etd/2120

This Thesis is brought to you for free and open access by the Graduate School at VCU Scholars Compass. It has been accepted for inclusion in Theses and Dissertations by an authorized administrator of VCU Scholars Compass.

For more information, please contact libcompass@vcu.edu. 
College of Humanities and Sciences

Virginia Commonwealth University

This is to certify that the thesis prepared by Heather C. Smith titled "Zero Divisors among Digraphs" has been approved by her committee as satisfactory completion of the thesis requirement for the degree of Master of Science.

Richand Hammack

Richard H, Hammack, College of Humanities and Sciences

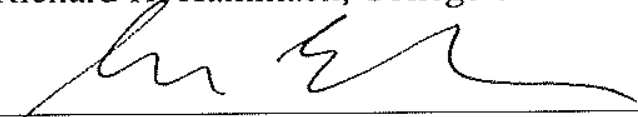

Craig E. Larson, College of Humanities and Sciences
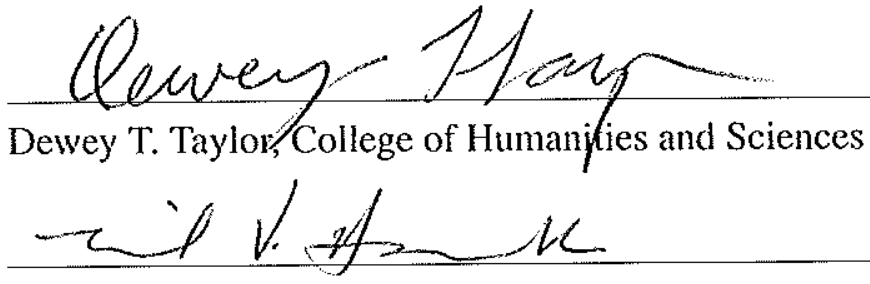

Micol V. Hammack, Department of Core Education

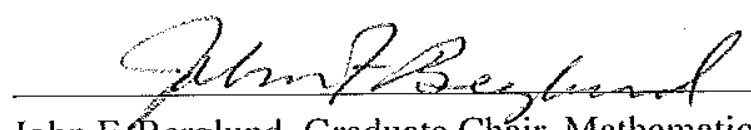

John F, Berglund, Graduate Chair, Mathematics and Applied Mathematics

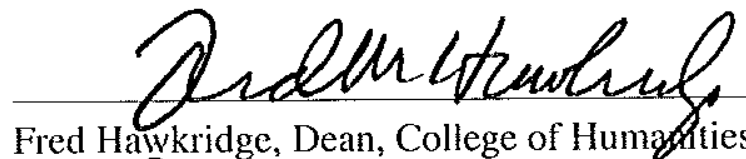

Fred Hawkridge, Dean, College of Humgrities and Sciences

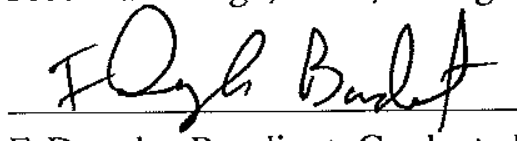

F. Douglas Boudinot, Graduate Dean

$$
\frac{\text { Apr:1 } 22,2010}{\text { Date }}
$$


(C) Heather C. Smith 2010

All Rights Reserved 


\section{Zero Divisors among Digraphs}

A thesis submitted in partial fulfillment of the requirements for the degree of Master of Science at Virginia Commonwealth University.

by

Heather C. Smith

Master of Science

Director: Richard H. Hammack, Associate Professor

Department of Mathematics and Applied Mathematics

Virginia Commonwealth University

Richmond, Virginia

May 2010 


\section{Acknowledgment}

I would like to thank Dr. Hammack for his great help through the development of this thesis. Dr. Hammack, thank you for your time, your patience, your attention to detail, and for sharing some of your knowledge with me. You always amaze me with the fresh new ideas you have. Thank you for your guidance this semester and for showing me how much fun graph theory can be. It has been wonderful working with you!

Most importantly, I would like to thank my Lord and Savior Jesus Christ who makes the beauty of math possible and who already knows the answer to any question we could ask. He just chooses when, where, and to whom to impart the knowledge to find a counterexample or complete a proof. I thank Him for sharing ideas with Dr. Hammack and for revealing proofs to me. 


\section{Contents}

Abstract $\quad$ iv

1 Introduction 1

1.1 Basic definitions from graph theory . . . . . . . . . . . . . 1

1.2 Mappings between digraphs . . . . . . . . . . . . . . . . 7

1.3 Extracting new digraphs from existing digraphs . . . . . . . . . 8

2 Survey of Known Results 11

2.1 Collections of homomorphisms . . . . . . . . . . . . . . . 11

2.2 Product graph isomorphisms . . . . . . . . . . . . . . . 16

2.3 Zero-divisors . . . . . . . . . . . . . . . . 17

3 Statement of Problem and Results 20

3.1 Zero-divisors ....................... 20

3.2 Automorphisms . . . . . . . . . . . . . . . . 22

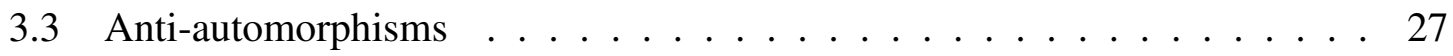

3.4 Factorial of a digraph . . . . . . . . . . . . . . . 32

3.5 Future directions . . . . . . . . . . . . . . . 39

4 Auxiliary Results 43

4.1 Additional results for zero-divisors . . . . . . . . . . . . . . . . . 43

4.2 Additional results for power graphs $A^{\alpha} \ldots \ldots \ldots$. . . . . . . . . . 44

$\begin{array}{ll}\text { Bibliography } & 49\end{array}$

$\begin{array}{lr}\text { Vita } & 50\end{array}$ 


\begin{abstract}
ZERO DIVISORS AMONG DIGRAPHS

By Heather C. Smith, Master of Science.

A thesis submitted in partial fulfillment of the requirements for the degree of Master of Science at Virginia Commonwealth University.

Virginia Commonwealth University, 2010.

Director: Richard H. Hammack, Associate Professor, Department of Mathematics and Applied Mathematics.
\end{abstract}

This thesis generalizes to digraphs certain recent results about graphs. There are special digraphs $C$ such that $A \times C \cong B \times C$ for some pair of distinct digraphs $A$ and $B$. Lovász named these digraphs $C$ zero-divisors and completely characterized their structure. Knowing that all directed cycles are zero-divisors, we focus on the following problem: Given any directed

cycle $\overrightarrow{C_{n}}$ and any digraph $A$, enumerate all digraphs $B$ such that $A \times \overrightarrow{C_{n}} \cong B \times \overrightarrow{C_{n}}$. From our result for cycles, we generalize to an arbitrary zero-divisor $C$, developing upper and lower bounds for the collection of digraphs $B$ satisfying $A \times C \cong B \times C$. 
Introduction

This thesis explores products of digraphs and their isomorphisms. Lovász first pointed out that there are certain digraphs $C$ for which you can find different digraphs $A$ and $B$ with $A \times C \cong B \times C$. From this property, he coined the term "zero-divisor" for such digraphs $C$. Examining these zero-divisors more closely, Lovász was able to completely characterize their structure. Working from a slightly different angle, our goal is to find all pairs $A$ and $B$ for zero-divisors of the form $\overrightarrow{C_{n}}$ such that $A \times \overrightarrow{C_{n}} \cong B \times \overrightarrow{C_{n}}$. We do this by fixing $A$ and determining the possibilities for $B$ based upon some of the properties of $A$. As found in chapter 3, we make use of power graphs $A^{\alpha}$ from automorphisms and anti-automorphisms $\alpha$. Then we generalize our theorem using the factorial $A$ !. From this digraph, we are able to fully characterize all $B$ given a digraph $A$ and a zero-divisor which is a cycle. Using another theorem from Lovász, we are able to develop upper and lower bounds for the collection of digraphs $B$ given any zero-divisor.

\subsection{Basic definitions from graph theory}

Let us pave the way for our results by first stating a few definitions that will be useful in later sections. We will start at the beginning with some basic terminology from graph theory. A graph is uniquely defined by its vertices and edges. The vertices are points typically denoted $v_{0}, v_{1}, \ldots, v_{n}$. Edges occur between pairs of vertices, connecting one to another. An edge between vertices $v_{i}$ and $v_{j}$ is written as an unordered pair $\left\{v_{i}, v_{j}\right\}$ or, for shorthand, $v_{i} v_{j}$ (which is the same as $v_{j} v_{i}$ ). The vertex set of a graph $G$ is denoted $V(G)$, while the 


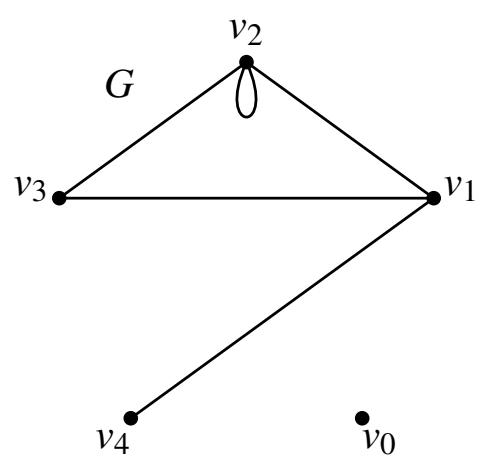

Figure 1.1: A graph $G$ with $V(G)=\left\{v_{0}, v_{1}, v_{2}, v_{3}, v_{4}\right\}$ and $E(G)=$ $\left\{v_{1} v_{2}, v_{2} v_{3}, v_{2} v_{2}, v_{3} v_{1}, v_{4} v_{1}\right\}$

edge set of $G$ is denoted $E(G)$. The cardinality of the vertex set is called the order of a graph. Similarly, the cardinality of the edge set is the size of a graph. A visual representation of a graph $G$ of order 5 and size 5 is drawn in Figure 1.1. You will notice that among the edges in $G$, there is an edge that begins and ends with $v_{2}$. Such an edge is called a loop. In addition, the vertex $v_{0}$ is not found in any edge pair. Such a vertex is referred to as an isolated vertex. Because graphs are uniquely determined by their vertices and edges, the spacial arrangement of the vertices is irrelevant.

A directed graph, more commonly referred to as a digraph, differs from a graph in that each edge is given a direction. Thus the edge $\left(v_{i}, v_{j}\right)$ begins at vertex $v_{i}$ and ends with vertex $v_{j}$. Directed edges, also known as arcs, are drawn with arrows to indicate direction. Note that, in a digraph, edges must be written as ordered pairs because the edges $v_{i} v_{j}$ and $v_{j} v_{i}$ are distinct based upon their direction. The edge $v_{i} v_{j}$ is the edge from vertex $v_{i}$ to vertex $v_{j}$ whereas the edge $v_{j} v_{i}$ will have an arrow pointing away from $v_{j}$ and toward $v_{i}$. Consider the representation of a typical digraph in Figure 1.2. Observe there is a loop on vertex $v_{2}$. In a digraph, the direction of the loop is not specified because it is symmetric with itself. Many times it is useful to consider only those graphs and digraphs without loops. We call this set $\Gamma$. If loops are allowed, we are looking at the larger set of graphs and digraphs $\Gamma_{0}$. 


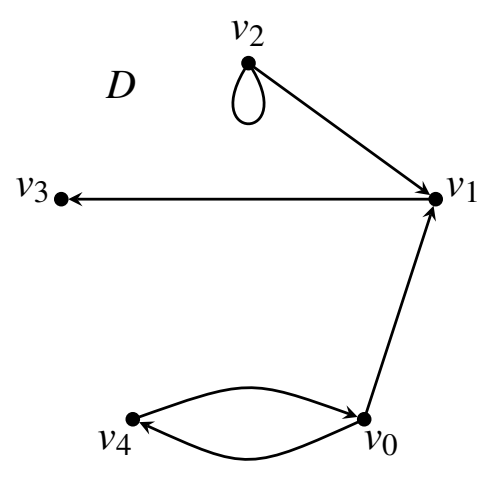

Figure 1.2: A digraph $D$ with $V(D)=\left\{v_{0}, v_{1}, v_{2}, v_{3}, v_{4}\right\}$ and $E(D)=$ $\left\{v_{2} v_{2}, v_{2} v_{1}, v_{1} v_{3}, v_{0} v_{1}, v_{4} v_{0}, v_{0} v_{4}\right\}$

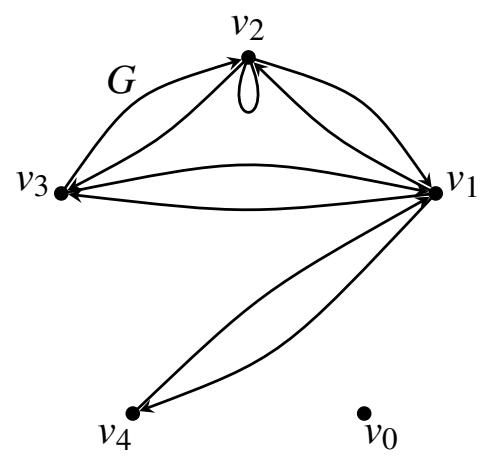

Figure 1.3: A symmetric digraph representation of $G$ from Figure 1.1

Every graph can be drawn as a symmetric digraph. For any edge $v_{i} v_{j}$ in a graph, the symmetric digraph counterpart would have corresponding arcs $v_{i} v_{j}$ and $v_{j} v_{i}$. Because both arcs are present for each edge in the graph, the digraph is considered symmetric. (See the digraph representation of graph $G$ in Figure 1.3.) So, graphs comprise a subset of digraphs.

Two vertices in a graph are said to be adjacent if there is an edge between them. For example, the edge $v_{1} v_{3}$ in $G$ from Figure 1.1 indicates that $v_{1}$ is adjacent to $v_{3}$ and $v_{3}$ is adjacent to $v_{1}$. We can also say that vertex $v_{1}$ is incident with the edge $v_{1} v_{3}$. The degree of a vertex is equal to the number of edges with which it is incident. Again in Figure 1.1, the degree of vertex $v_{1}$ in $G$ is 3 . 
Keep in mind that the direction of an edge in a digraph is important. In the digraph $D$ from Figure 1.2, the edge $v_{1} v_{3}$ exists, but $v_{3} v_{1}$ is not an edge. So $v_{1}$ is said to be adjacent to $v_{3}$, but $v_{3}$ is not adjacent to $v_{1}$. Because of the direction of edges in a digraph, here we talk more specifically about the indegree and outdegree of vertices. The indegree of a vertex $v_{i}$ is equal to the number of edges with the arrows pointing away from $v_{i}$, whereas the outdegree of a vertex $v_{i}$ is equal to the number of edges with arrows pointing toward $v_{i}$. Referencing $D$ in Figure 1.2, the indegree of $v_{1}$ is 2 and the outdegree of $v_{1}$ is 1 .

From the adjacencies in a digraph, we can define a walk.

DEFINITION 1.1. A walk in a digraph $A$ is a finite sequence of vertices $v_{0} v_{1} v_{2} \cdots v_{n}$ such that $v_{i} v_{i+1}$ is an edge for all $i \in\{0,1,2, \ldots, n-1\}$. Within the sequence, vertices may be repeated. The length of a walk is equal to the number of edges traversed while going from $v_{0}$ to $v_{n}$, thus $n$. A closed walk is a walk that begins and ends with the same vertex.

For instance, $v_{1} v_{4} v_{1} v_{2} v_{2} v_{3}$ is a walk of length 5 in $G$ as seen in Figure 1.3. A closed walk of length 3 in $G$ could be represented by $v_{1} v_{2} v_{3} v_{1}$. A path is a type of walk where no vertex is repeated. So $v_{4} v_{1} v_{2} v_{3}$ is a path of length 3 in $G$ in Figure 1.3.

Now we can define connectedness and connected components.

DEFINITION 1.2. A graph is connected if and only if there is a sequence of edges between every pair of vertices that can be traversed either forwards or backwards to travel from one vertex to the other.

DEFINITION 1.3. A connected component of a graph or digraph is a maximal connected subgraph (a subset of the vertices from the original graph together with any edges among them).

A connected digraph and a disconnected digraph with 3 components can be found in Figure 1.4. You may notice, in $G$ there is path from 0 to 4 . This path consists of the edges 

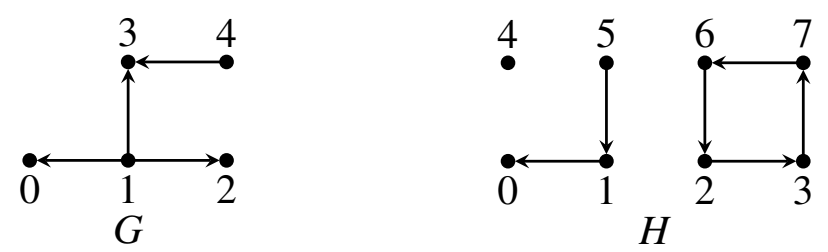

Figure 1.4: A connected digraph $G$ and a disconnected digraph $H$ with 3 components, $\{4\},\{0,1,5\},\{2,3,6,7\}$

$\{10,13,43\}$. This collection of edges, though not forming a directed path, does provide a connection between vertices 0 and 4 . Because some collection of edges exists between every pair of vertices in $G$, we say $G$ is connected. In $H$, there is no connection between vertices 1 and 2, so $H$ is disconnected. However, we can identify the components of $H$ by looking for connected subgraphs. The vertices in the set $\{2,3,6,7\}$ and the edges among them form a connected component, as do the vertices in $\{0,1,5\}$. By definition, the isolated vertex 4 is itself a connected component. Vacuously, it is connected and there is no other vertex in $H$ that could be included without causing the subgraph to be disconnected. So it is a maximal connected subgraph, and thus a component of $H$.

Cycles make up a special class of graphs with directed cycles being their analogues in digraphs.

Definition 1.4. A graph is a cycle if it is connected and the degree of every vertex is two. A cycle of length $n$, where $n$ is the number of edges in the cycle, is denoted $C_{n}$. A digraph is a directed cycle if the outdegree and indegree of every vertex is one. Such a digraph on $n$ vertices is denoted $\overrightarrow{C_{n}}$.

For consistency, we will label the vertices of a cycle $C_{n}$ or directed cycle $\overrightarrow{C_{n}}$ with $\{0,1,2, \ldots, n-1\}$ consecutively so that $\{01,12, \ldots,(n-1)(0)\}$ are edges. Figure 1.5 gives an example of a cycle and a directed cycle. 

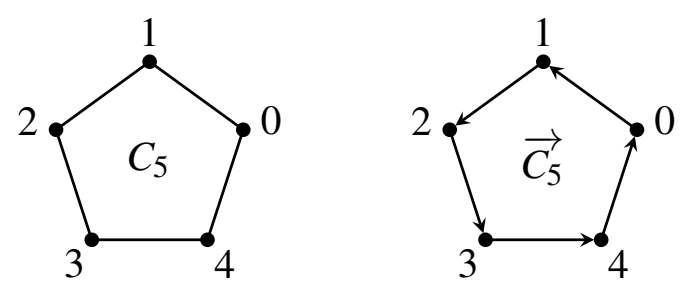

Figure 1.5: A cycle $C_{5}$ and a directed cycle $\overrightarrow{C_{5}}$
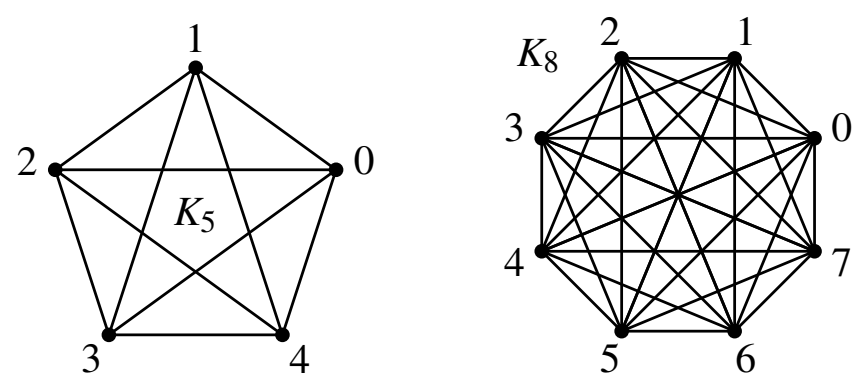

Figure 1.6: Examples of complete graphs on 5 and 8 vertices

Another special class of graphs are the complete graphs.

DEFINITION 1.5. A complete graph has every vertex adjacent to every other vertex. A complete graph on $n$ vertices is denoted $K_{n}$.

A couple examples of complete graphs are found in Figure 1.6. A few special cases of cycles and complete graphs, drawn as digraphs, are presented in Figure 1.7.

Given two distinct graphs, we can combine them in many different ways to create a new graph. One way is to take the product of the graphs. In particular, this paper focuses on the

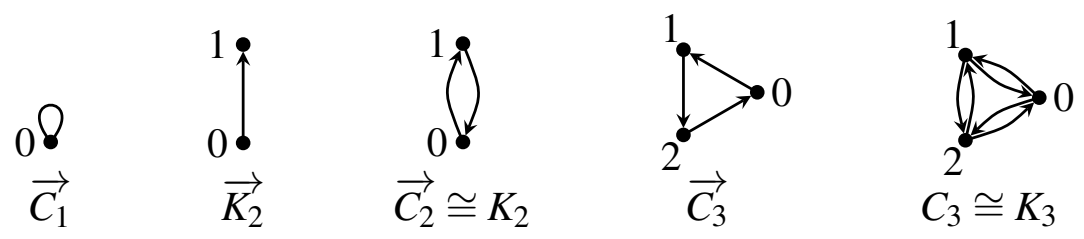

Figure 1.7: A few special cases of cycles and complete graphs 


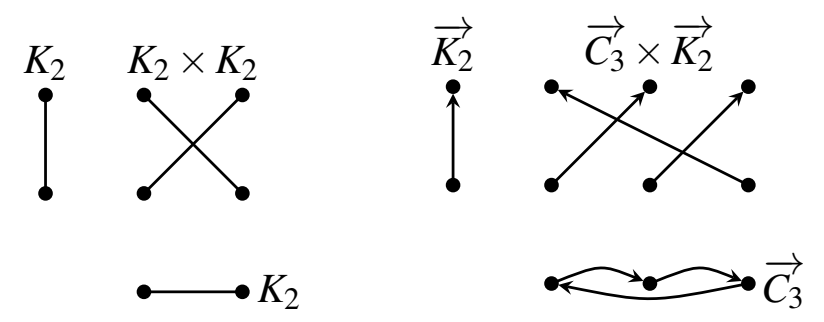

Figure 1.8: The direct product of graphs and digraphs

direct product. The direct product of two digraphs, $A$ and $B$, is denoted $A \times B$.

Definition 1.6. The direct product $A \times B$ is defined as follows:

$$
\begin{gathered}
V(A \times B)=\{(a, b): a \in V(A) \text { and } b \in V(B)\} \\
E(A \times B)=\left\{(a, b)\left(a^{\prime}, b^{\prime}\right): a a^{\prime} \in E(A) \text { and } b b^{\prime} \in E(B)\right\}
\end{gathered}
$$

A couple examples of direct products can be found in Figure 1.8. Interestingly, you may notice that the direct product of $K_{2}$ with itself is exactly the symbol for the direct product.

\subsection{Mappings between digraphs}

As was described in the abstract, our intent is to work with isomorphic direct products of graphs and digraphs whose factors may not be isomorphic. In order to develop the idea of an isomorphism, we first need the concept of a function between graphs and subsequently homomorphisms. We can define a function $\psi$ from one graph (or digraph) $A$ to another $B$ by mapping the vertices in $A$ to vertices in $B$. A homomorphism is a special type of function.

DEFInITION 1.7. A function $\psi: V(A) \rightarrow V(B)$ is a homomorphism if it preserves adjacencies. In other words, if $a a^{\prime}$ is an edge in $A$, then $\psi(a) \psi\left(a^{\prime}\right)$ must be an edge in $B$. 

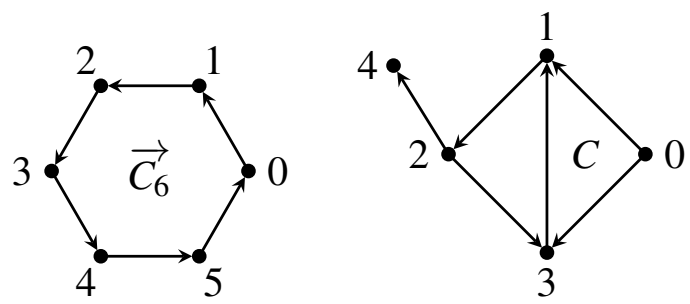

Figure 1.9: There is a homomorphism $\psi: V\left(\overrightarrow{C_{6}}\right) \rightarrow V(C)$

For the digraphs in Figure 1.9, there is a homomorphism $\psi: V\left(\overrightarrow{C_{6}}\right) \rightarrow V(C)$ defined by

$$
\begin{array}{lll}
\psi(0)=1 & \psi(1)=2 & \psi(2)=3 \\
\psi(3)=1 & \psi(4)=2 & \psi(5)=3
\end{array}
$$

More specific than homomorphisms are isomorphisms.

DEFINITION 1.8. A map $\psi: V(G) \rightarrow V(H)$ is an isomorphism if $\psi$ is a bijection and a homomorphism, and $\psi^{-1}$ is also a homomorphism. If such a function exists, then we say $G$ and $H$ are isomorphic or $G \cong H$.

In Figure 1.10, you will see two products that are isomorphic. The isomorphism $\psi: A \times K_{2} \rightarrow B \times K_{2}$ is defined as follows.

$$
\begin{aligned}
& \psi(1,1)=(1,1) \quad \psi(2,1)=(3,1) \quad \psi(3,1)=(2,1) \\
& \psi(1,0)=(1,0) \quad \psi(2,0)=(2,0) \quad \psi(3,0)=(3,0)
\end{aligned}
$$

\subsection{Extracting new digraphs from existing digraphs}

There are many ways of extracting a new digraph from an existing one by focusing on some particular characteristic or imposing an additional structure on the digraph. 

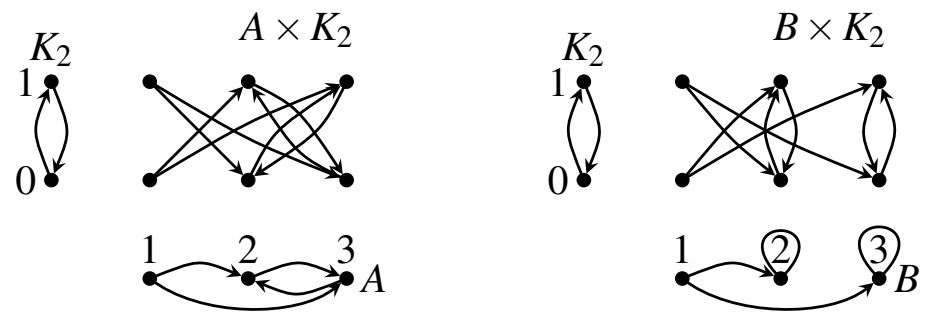

Figure 1.10: Two products $A \times K_{2}$ and $B \times K_{2}$ that are isomorphic

As we work toward ennumerating all digraphs $B$ such that $A \times C \cong B \times C$, the power graphs $A^{\mu}$ will be essential. The definition of $A^{\mu}$, a digraph extracted from $A$, is given below as presented in [3].

Definition 1.9. Given a permutation $\mu$ on the vertices of $\mathrm{A}$, the power graph $A^{\mu}$ is defined as follows:

$$
\begin{gathered}
V\left(A^{\mu}\right)=V(A) \\
E\left(A^{\mu}\right)=\left\{a \mu\left(a^{\prime}\right): a a^{\prime} \in E(A)\right\}
\end{gathered}
$$

Recall a permutation on the vertices of $A$ is a bijection from $V(A)$ to $V(A)$.

An illustration of one such digraph, that will appear in later sections, is found in Figure 1.11. Observe (23) is a permutation of the vertices of $A$, mapping $1 \rightarrow 1,2 \rightarrow 3$, and $3 \rightarrow 2$.
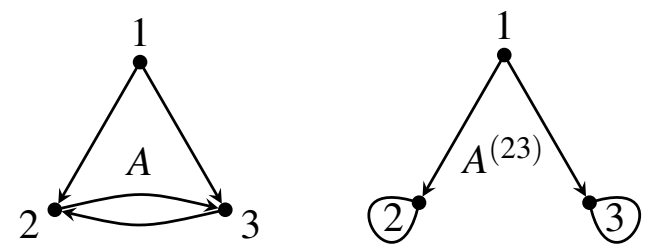

Figure 1.11: A digraph $A$ and permutation (23) giving $A^{(23)}$ 
Then, the edges in $A^{(23)}$ are found as follows:

$$
\begin{array}{ll}
12 \in E(A) \Longleftrightarrow 13 \in E\left(A^{(23)}\right) & 13 \in E(A) \Longleftrightarrow 12 \in E\left(A^{(23)}\right) \\
23 \in E(A) \Longleftrightarrow 22 \in E\left(A^{(23)}\right) & 32 \in E(A) \Longleftrightarrow 33 \in E\left(A^{(23)}\right)
\end{array}
$$

We can also define a quotient graph for $A$ given a partition of its vertices. Recall a partition on the vertices of $A$ is a collection of subsets of $V(A)$, say $A_{1}, A_{2}, \ldots, A_{n}$, such that the subsets are pairwise disjoint, but their union is the entire set $V(A)$. The following definition is based upon [1].

DEFINITION 1.10. For any digraph $A$, given a partition $\Omega=\left\{A_{1}, A_{2}, \ldots, A_{n}\right\}$ on $V(A)$, the quotient digraph $A / \Omega$ is defined as follows:

$$
\begin{gathered}
V(A / \Omega)=\left\{A_{1}, A_{2}, \ldots, A_{n}: A_{i} \in \Omega \text { for all } i\right\}=\Omega \\
E(A / \Omega)=\left\{A_{i} A_{j}: \exists u \in A_{i} \text { and } \exists v \in A_{j} \text { with } u v \in E(A) \text { for } A_{i}, A_{j} \in \Omega\right\}
\end{gathered}
$$

For example, consider the digraph $A$ and partition $\Omega=\{\{1,2,5,6\},\{3\},\{7\},\{4,8\}\}$ in Figure 1.12. From these parameters, we develop the quotient graph $A / \Omega$. For example, the edge $(\{3\})(\{4,8\})$ exists in the quotient because $(3)(4)$ is an edge in $A$. The loop on $\{4,8\}$ in the quotient graph results from the edge $(4)(8)$ in $A$.
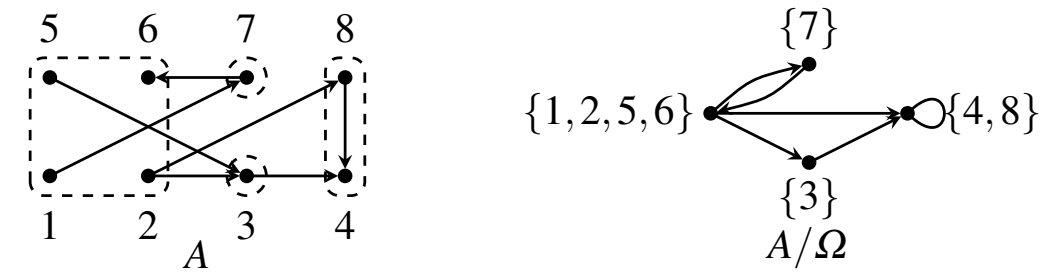

Figure 1.12: A digraph $A$ with partition $\Omega=\{\{1,2,5,6\},\{3\},\{7\},\{4,8\}\}$ and the corresponding quotient graph $A / \Omega$ 


\section{Survey of Known Results}

This chapter includes some existing results and corresponding proofs that will be referenced to in Chapter 3.

\subsection{Collections of homomorphisms}

These first three results pertain to the collection of homomorphisms between pairs of digraphs. Recall, a homomorphism from one digraph $X$ to another $A$ is a function from the vertices of $X$ to the vertices of $A$ that preserves adjacencies. There are often several different homomorphisms between two digraphs. The collection of these homomorphisms forms the set $\operatorname{Hom}(X, A)$. Then, the cardinality of this set is denoted $\operatorname{hom}(X, A)$. We begin with a lemma that will be useful in the next theorem. The proof for Lemma 2.1 for homomorphisms is based upon a proof for weak homomorphisms given in [1].

Lemma 2.1. For digraphs $X$ and $A$, let $\mathscr{P}$ be the set of all partitions of $V(X)$ and let $\operatorname{inj}(X, A)$ be the number of injective homomorphisms $X \rightarrow A$. Then

$$
\operatorname{hom}(X, A)=\sum_{\Omega \in \mathscr{P}} \operatorname{inj}(X / \Omega, A)
$$

where quotients are taken in $\Gamma_{0}$. 
Proof. We begin by defining two sets. Let $\operatorname{Hom}(X, A)$ denote the collection of all homomorphisms $X \rightarrow A$. So $|\operatorname{Hom}(X, A)|=\operatorname{hom}(X, A)$. Let

$$
\Upsilon=\left\{\left(\Omega, f^{*}\right): \Omega \in \mathscr{P}, f^{*} \text { is an injective homomorphism } X / \Omega \rightarrow A\right\} .
$$

Looking closely at this collection $\Upsilon$, one can see that for any given partition $\Omega^{\prime}$, there is a pair $\left(\Omega^{\prime}, f^{*}\right)$ for each injective homomorphism $f^{*}: X / \Omega^{\prime} \rightarrow A$. So $|\Upsilon|=\sum_{\Omega \in \mathscr{P}} \operatorname{inj}(X / \Omega, A)$.

In order to prove the equality $\operatorname{hom}(X, A)=\sum_{\Omega \in \mathscr{P}} \operatorname{inj}(X / \Omega, A)$, we will develop a bijection $\beta: \operatorname{Hom}(X, A) \rightarrow \Upsilon$. Given any homomorphism $f: X \rightarrow A$, the function $f$ creates a partition on the vertices of $X$, specifically $\Omega_{f}=\left\{f^{-1}(a): a \in V(A)\right\}$. We can correlate this to an injective homomorphism $f^{*}: X / \Omega_{f} \rightarrow A$ where, for any $U \in \Omega_{f}$, the function $f^{*}$ is defined as $f^{*}(U)=f(u)$ for any $u \in U$. So our mapping $\beta: \operatorname{Hom}(X, A) \rightarrow \Upsilon$ will be defined by $\beta(f)=\left(\Omega_{f}, f^{*}\right)$ as described above.

Let us prove $\beta$ is a bijection to complete the proof. First, for any $\left(\Omega, f^{*}\right) \in \Upsilon$, let $f(u)=f^{*}(U)$ for all $u \in U \in \Omega$. Then $f: X \rightarrow A$ is a homomorphism because $f^{*}: X / \Omega \rightarrow A$ is a homomorphism. Specifically, for any edge $u v$ in $X$, if $u \in U$ and $v \in V$ where $U$ and $V$ are different sets in $\Omega$, then clearly $f(u) f(v)=f^{*}(U) f^{*}(V)$ is an edge in $A$. If both $u$ and $v$ come from the same set $U$, then $X / \Omega$ must have a loop on vertex $U$. So $f^{*}(U)$ must also have a loop since $f^{*}$ is a homomorphism. Then $f(u) f(v)=f^{*}(U) f^{*}(U)$ which is in fact an edge in $A$. So $f$ is a homomorphism in $\operatorname{Hom}(X, A)$ that maps to $\left(\Omega, f^{*}\right)$ allowing us to conclude that $\beta$ is surjective. Second, for any two functions $f, g \in \operatorname{Hom}(X, A)$, suppose $\beta(f)=\beta(g)$. So $\left(\Omega_{f}, f^{*}\right)=\left(\Omega_{g}, g^{*}\right)$ giving us the same partition on $V(X)$ and $f^{*}=g^{*}$. In this case, for any $U \in \Omega_{f}$ and any $u \in U$, we conclude $f(u)=f^{*}(U)=g^{*}(U)=g(u)$. So $f=g$ making $\beta$ injective. Therefore our function $\beta: \operatorname{Hom}(X, A) \rightarrow \Upsilon$ is a bijection implying 


$$
\operatorname{hom}(X, A)=|\operatorname{Hom}(X, A)|=|\Upsilon|=\sum_{\Omega \in \mathscr{P}} \operatorname{inj}(X / \Omega, A)
$$

completing the proof.

The following theorem and subsequent proof are based on [1] and [3] but have been rephrased for this thesis.

THEOREM 2.2. If $G$ and $H$ are digraphs, then $G \cong H$ if and only if $\operatorname{hom}(X, G)=\operatorname{hom}(X, H)$ for every digraph $X$.

Proof. Let $G$ and $H$ be digraphs. First assume that $G \cong H$. Then there exists a bijective homomorphism $\psi: G \rightarrow H$ such that $\psi^{-1}: H \rightarrow G$ is also a homomorphism. Then observe for any homomorphism $\phi: X \rightarrow G$, the composition $\psi \phi: X \rightarrow H$ is a homomorphism. So $\operatorname{hom}(X, G) \leq \operatorname{hom}(X, H)$. Likewise, for any homomorphism $\mu: X \rightarrow H$, the composition $\psi^{-1} \mu: X \rightarrow G$ is a homomorphism. So $\operatorname{hom}(X, H) \leq \operatorname{hom}(X, G)$. And finally, we have the equality $\operatorname{hom}(X, G)=\operatorname{hom}(X, H)$.

Conversely, assume $\operatorname{hom}(X, G)=\operatorname{hom}(X, H)$ for every digraph $X$. We will show $\operatorname{inj}(X, G)=\operatorname{inj}(X, H)$ for all digraphs $X$. Using this and the fact that identity maps are injective homomorphisms, we have $1 \leq \operatorname{inj}(G, G)=\operatorname{inj}(G, H)$ and $\operatorname{inj}(H, G)=\operatorname{inj}(H, H) \geq 1$. Thus there exists an injective homomorphism $\psi: G \rightarrow H$ giving $|V(G)| \leq|V(H)|$ and $|E(G)| \leq|E(H)|$. And there is an injective homomorphism $\mu: H \rightarrow G$ which gives $|V(G)| \geq|V(H)|$ and $|E(G)| \geq|E(H)|$. So $|V(G)|=|V(H)|$ and $|E(G)|=|E(H)|$. Thus $\psi: G \rightarrow H$ must be surjective. So we have a bijective homomorphism between $G$ and $H$. Suppose there is an edge $x y$ in $H$ where $\psi^{-1}(x) \psi^{-1}(y)$ is not an edge in $G$. Because $\psi$ is an injective homomorphism, every edge in $G$ maps to a unique edge in $H$. With the existence of this edge $x y$, we are saying there is at least one more edge in $H$ than in $G$, which contradicts our earlier conclusion that $|E(G)|=|E(H)|$. So $\psi^{-1}$ must be homomorphism. And finally, by definition, $\psi$ is an isomorphism, so $G \cong H$. 
In order to prove $\operatorname{inj}(X, G)=\operatorname{inj}(X, H)$, we will induct on the number of vertices in $X$, $|V(X)|$. First, observe for $|V(X)|=1$, every homomorphism from $X$ to another digraph is injective because $X$ has only a single vertex. Then by our earlier assumption that $\operatorname{hom}(X, G)=\operatorname{hom}(X, H)$, we have

$$
\operatorname{inj}(X, G)=\operatorname{hom}(X, G)=\operatorname{hom}(X, H)=\operatorname{inj}(X, H) .
$$

Suppose for every $X$ with fewer than $n$ vertices, $\operatorname{inj}(X, G)=\operatorname{inj}(X, H)$. Now consider a digraph $X$ with order $n$. Let $\imath \in \mathscr{P}$ be the trivial partition where each class consists of a single vertex. So the quotient graph $X / \imath$ is isomorphic to $X$. But for any other partition $\Omega \in \mathscr{P} \backslash \imath$, there will be at least one class with more than one vertex, so $|V(X / \Omega)|<n$. Recall from Lemma 2.1, hom $(X, G)=\sum_{\Omega \in \mathscr{P}} \operatorname{inj}(X / \Omega, G)$. By separating the partition $\imath$ from the rest, we get $\operatorname{hom}(X, G)=\operatorname{inj}(X / \imath, G)+\sum_{\Omega \in \mathscr{P} \backslash \imath} \operatorname{inj}(X / \Omega, G)$ which by our induction hypothesis equals $\operatorname{inj}(X, G)+\sum_{\Omega \in \mathscr{P} \backslash l} \operatorname{inj}(X / \Omega, H)$. Likewise,

$$
\operatorname{hom}(X, H)=\sum_{\Omega \in \mathscr{P}} \operatorname{inj}(X / \Omega, H)=\operatorname{inj}(X, H)+\sum_{\Omega \in \mathscr{P} \backslash \imath} \operatorname{inj}(X / \Omega, H)
$$

By our original assumption, $\operatorname{hom}(X, G)=\operatorname{hom}(X, H)$, so

$$
\operatorname{inj}(X, G)+\sum_{\Omega \in \mathscr{P} \backslash \iota} \operatorname{inj}(X / \Omega, H)=\operatorname{inj}(X, H)+\sum_{\Omega \in \mathscr{P} \backslash \iota} \operatorname{inj}(X / \Omega, H)
$$

And finally, $\operatorname{inj}(X, G)=\operatorname{inj}(X, H)$. By the above argument, the proof is complete.

The next theorem and proof are based on a proposition and proof in [1] for strong products, but they have been reworked for direct products. 
THEOREM 2.3. If $X, G$, and $H$ are digraphs, then

$$
\operatorname{hom}(X, G \times H)=\operatorname{hom}(X, G) \cdot \operatorname{hom}(X, H) \text {. }
$$

Proof. Let $X, G$, and $H$ be digraphs. Let $\operatorname{Hom}(X, G \times H)$ be the set of homomorphisms from $X$ to $G \times H$. Likewise, the sets $\operatorname{Hom}(X, G)$ and $\operatorname{Hom}(X, H)$ will be the collections of homomorphisms from $X$ to $G$ and to $H$, respectively. In order to prove the desired result $\operatorname{hom}(X, G \times H)=\operatorname{hom}(X, G) \cdot \operatorname{hom}(X, H)$, we will define a bijection from the collection of functions $\operatorname{Hom}(X, G \times H)$ to the Cartesian product of functions $\operatorname{Hom}(X, G) \times \operatorname{Hom}(X, H)$ giving us equal cardinalities and completing our proof.

First, observe there are natural homomorphisms $\pi_{G}: V(G \times H) \rightarrow V(G)$ defined as $\pi_{G}(g, h)=g$ and $\pi_{H}: V(G \times H) \rightarrow V(H)$ with $\pi_{H}(g, h)=h$ called projections. Keeping this in mind, define

$$
\mu: \operatorname{Hom}(X, G \times H) \rightarrow \operatorname{Hom}(X, G) \times \operatorname{Hom}(X, H)
$$

as $\mu(f)=\left(\pi_{G} f, \pi_{H} f\right)$. Suppose there are two homomorphisms $f, g \in \operatorname{Hom}(X, G \times H)$ with $\mu(f)=\mu(g)$. Then $\left(\pi_{G} f, \pi_{H} f\right)=\left(\pi_{G} g, \pi_{H} g\right)$ and consequently $\pi_{G} f=\pi_{G} g$ giving us $f=g$. So $\mu$ is injective. Next let $(k, l) \in \operatorname{Hom}(X, G) \times \operatorname{Hom}(X, H)$. Define a function $f: V(X) \rightarrow V(G \times H)$ as $f(x)=(k(x), l(x))$. Observe because $k$ and $l$ are homomorphisms, $k(x y)=k(x) k(y)$ is an edge in $G$ and $l(x y)=l(x) l(y)$ is an edge in $\mathrm{H}$, so $f(x y)=(k(x), l(x))(k(y), l(y))$ is an edge in $G \times H$ by the construction of edges in a direct product. So $f$ is a homomorphism in $\operatorname{Hom}(X, G \times H)$. Then observe that $\pi_{G} f=k$ and $\pi_{H} f=l$, so $\mu(f)=(k, l)$ and $\mu$ is surjective. 
Because $\mu$ is a bijection, the cardinalities of $\operatorname{Hom}(X, G \times H)$ and $\operatorname{Hom}(X, G) \times \operatorname{Hom}(X, H)$ are equal. $\operatorname{So} \operatorname{hom}(X, G \times H)=\operatorname{hom}(X, G) \cdot \operatorname{hom}(X, H)$.

\subsection{Product graph isomorphisms}

As we lay the foundation for working with isomorphic direct products, the following theorems of Lovász will be beneficial. Recall the following Theorem of Lovász from [4]. Our proof takes a more direct approach.

Theorem 2.4. (Lovász [4], Theorem 6) Let $A, B, C$ and $D$ be digraphs. If $A \times C \cong B \times C$ and there is a homomorphism from $D$ to $C$, then $A \times D \cong B \times D$.

Proof. Suppose $A \times C \cong B \times C$ and there is a homomorphism from $D$ to $C$ where $A, B, C$ and $D$ are digraphs. Then by Theorem $2.2, \operatorname{hom}(X, A \times C)=\operatorname{hom}(X, B \times C)$ for any digraph $X$. And by Theorem 2.3, $\operatorname{hom}(X, A) \operatorname{hom}(X, C)=\operatorname{hom}(X, B) \operatorname{hom}(X, C)$. Consider two cases: (1) $\operatorname{hom}(X, C)=0$, and (2) $\operatorname{hom}(X, C) \neq 0$.

Case (1): If $\operatorname{hom}(X, C)=0$ then $\operatorname{hom}(X, D)=0$ since, for any homomorphism from $X$ to $D$, the composition of this with the homomorphism from $D$ to $C$ is itself a homomorphism from $X$ to $C$. Thus $\operatorname{hom}(X, C)=\operatorname{hom}(X, D)=0$, so

$$
\operatorname{hom}(X, A) \operatorname{hom}(X, D)=\operatorname{hom}(X, B) \operatorname{hom}(X, D) \text {. }
$$

By Theorem 2.3, this implies that $\operatorname{hom}(X, A \times D)=\operatorname{hom}(X, B \times D)$ for all $X$ such that $\operatorname{hom}(X, C)=0$.

Case (2) If $\operatorname{hom}(X, C) \neq 0$ then $\operatorname{hom}(X, A) \operatorname{hom}(X, C)=\operatorname{hom}(X, B) \operatorname{hom}(X, C)$ becomes $\operatorname{hom}(X, A)=\operatorname{hom}(X, B)$. Now, we can multiply both sides by $\operatorname{hom}(X, D)$ giving us the equation

$$
\operatorname{hom}(X, A) \operatorname{hom}(X, D)=\operatorname{hom}(X, B) \operatorname{hom}(X, D) \text {. }
$$


So by Theorem 2.3, this implies that $\operatorname{hom}(X, A \times D)=\operatorname{hom}(X, B \times D)$ for all $X$ such that $\operatorname{hom}(X, C) \neq 0$.

Together, Cases (1) and (2) show that for all $X$, $\operatorname{hom}(X, A \times D)=\operatorname{hom}(X, B \times D)$. Finally, by Theorem $2.2, A \times D \cong B \times D$.

Lovász presented Theorem 2.5 which can greatly simplify the structure of isomorphisms between product graphs. It may prove helpful to reexamine Figure 1.10 and the isomorphism $\psi$ presented in the previous chapter in order to better understand this theorem.

Theorem 2.5. (Lovász [4], Theorem 7) Let $A, B$, and $C$ be digraphs. If $A \times C \cong B \times C$ then there is an isomorphism $\Psi: V(A \times C) \rightarrow V(B \times C)$ with $\Psi(a, c)=\left(\beta_{c}(a), c\right)$ for homomorphisms $\beta_{c}: V(A \times B) \rightarrow V(C)$ for each $c \in V(C)$.

\subsection{Zero-divisors}

The following theorem and subsequent corollary are the main results of [5]. As will be seen in the next chapter, our work seeks to extend this result from $\overrightarrow{K_{2}}$ to directed cycles and then to any given zero-divisor.

THEOREM 2.6. For any digraph $A$, we have $A \times \overrightarrow{K_{2}} \cong B \times \overrightarrow{K_{2}}$ if and only if $B \cong A^{\alpha}$ for some permutation $\alpha$ on $V(A)$.

Proof. Given a digraph $A$, suppose first $A \times \overrightarrow{K_{2}} \cong B \times \overrightarrow{K_{2}}$. So, there is an isomorphism $\psi: V\left(A \times \overrightarrow{K_{2}}\right) \rightarrow V\left(B \times \overrightarrow{K_{2}}\right)$. By Theorem 2.5, this isomorphism can be expressed as $\psi(a, i)=\left(\beta_{i}(a), i\right)$ where $i \in\{0,1\}$. Thus the functions $\beta_{0}$ and $\beta_{1}$ are bijections from $V(A)$ to $V(B)$. Observe $\pi=\beta_{0}^{-1} \beta_{1}$ is a permutation on $V(A)$. Now consider the digraph $A^{\pi}$. In order to show that $A^{\pi} \cong B$, we develop a isomorphism from $V\left(A^{\pi}\right)$ to $V(B)$. 
Because $V(A)=V\left(A^{\pi}\right)$, we have a bijection $\beta_{0}: V\left(A^{\pi}\right) \rightarrow V(B)$. In order to show that $\beta_{0}$ and $\beta_{0}^{-1}$ are homomorphisms, consider the following equivalences:

$$
\begin{aligned}
a a^{\prime} \in E\left(A^{\pi}\right) & \Longleftrightarrow a \pi^{-1}\left(a^{\prime}\right) \in E(A) \\
& \Longleftrightarrow a \beta_{1}^{-1} \beta_{0}\left(a^{\prime}\right) \in E(A) \\
& \Longleftrightarrow(a, 0)\left(\beta_{1}^{-1} \beta_{0}\left(a^{\prime}\right), 1\right) \in E\left(A \times \overrightarrow{K_{2}}\right) \\
& \Longleftrightarrow \psi(a, 0) \psi\left(\beta_{1}^{-1} \beta_{0}\left(a^{\prime}\right), 1\right) \in E\left(B \times \overrightarrow{K_{2}}\right) \\
& \Longleftrightarrow\left(\beta_{0}(a), 0\right)\left(\beta_{1} \beta_{1}^{-1} \beta_{0}\left(a^{\prime}\right), 1\right) \in E\left(B \times \overrightarrow{K_{2}}\right) \\
& \Longleftrightarrow\left(\beta_{0}(a), 0\right)\left(\beta_{0}\left(a^{\prime}\right), 1\right) \in E\left(B \times \overrightarrow{K_{2}}\right) \\
& \Longleftrightarrow \beta_{0}(a) \beta_{0}\left(a^{\prime}\right) \in E(B) .
\end{aligned}
$$

So $\beta_{0}$ and $\beta_{0}^{-1}$ are both homomorphisms by the equivalences above. Thus $\beta_{0}$ is an isomorphism, allowing us to conclude $B \cong A^{\pi}$ for some permutation $\pi$ on $V(A)$.

Conversely, assume $B \cong A^{\pi}$ for some permutation $\pi$ on $V(A)$. We will show $A \times \overrightarrow{K_{2}}$ is isomorphic to $A^{\pi} \times \overrightarrow{K_{2}}$ by developing an isomorphism $\psi: V\left(A \times \overrightarrow{K_{2}}\right) \rightarrow V\left(A^{\pi} \times \overrightarrow{K_{2}}\right)$. Define $\psi$ as follows:

$$
\Psi(a, \varepsilon)= \begin{cases}(a, \varepsilon) & \text { if } \varepsilon=0 \\ (\pi(a), \varepsilon) & \text { if } \varepsilon=1\end{cases}
$$

Clearly $\psi$ is a bijection because $\pi$ is a permutation. Now we show that $\psi$ and $\psi^{-1}$ are homomorphisms. Let $(a, 0)\left(a^{\prime}, 1\right)$ be an arbitrary edge in $A \times \overrightarrow{K_{2}}$. Observe:

$$
\begin{aligned}
(a, 0)\left(a^{\prime}, 1\right) \in E\left(A \times \overrightarrow{K_{2}}\right) & \Longleftrightarrow a a^{\prime} \in E(A) \\
& \Longleftrightarrow a \pi\left(a^{\prime}\right) \in E\left(A^{\pi}\right) \\
& \Longleftrightarrow(a, 0)\left(\pi\left(a^{\prime}\right), 1\right) \in E\left(A^{\pi} \times \overrightarrow{K_{2}}\right) \\
& \Longleftrightarrow \psi(a, 0) \psi\left(a^{\prime}, 1\right) \in E\left(A^{\pi} \times \overrightarrow{K_{2}}\right)
\end{aligned}
$$


So $\psi$ and $\psi^{-1}$ are in fact homomorphisms, showing that $\psi$ is an isomorphism. Thus we may conclude $A \times \overrightarrow{K_{2}} \cong A^{\pi} \times \overrightarrow{K_{2}}$.

COROLLARY 2.7. Given any zero-divisor $C$ with a surjective homomorphism onto $\overrightarrow{K_{2}}$ and any digraph $A$, then $A \times C \cong B \times C$ if and only if $B \cong A^{\alpha}$ for some permutation $\alpha$ on $V(A)$.

Proof. Let $C$ be a zero-divisor with a surjective homomorphism onto $\overrightarrow{K_{2}}$. First, assume $A \times C \cong B \times C$. Because we have a surjective map $C \rightarrow \overrightarrow{K_{2}}$, the digraph $C$ has at least one edge. Thus, there is a homomorphism $\overrightarrow{K_{2}} \rightarrow C$. By Theorem 2.4, we are guaranteed $A \times \overrightarrow{K_{2}} \cong B \times \overrightarrow{K_{2}}$. And by Theorem $2.6, B$ is necessarily isomorphic to $A^{\alpha}$ for some permutation $\alpha$ on the vertices of $A$.

Conversely assume $B \cong A^{\alpha}$ for some permutation $\alpha$ on $V(A)$. Then by Theorem 2.6, $A \times \overrightarrow{K_{2}} \cong A^{\alpha} \times \overrightarrow{K_{2}}$. Because there is a homomorphism $C \rightarrow \overrightarrow{K_{2}}$, by Theorem 2.4, $A \times C \cong B \times C$ and the proof is complete.

This completes our review of standing results, many of which are attributed to the work of Lovász. Keep these results in mind as we will employ many of them in the upcoming chapter. 


\section{Statement of Problem and Results}

The purpose and goal of this thesis is to examine isomorphisms of the form $A \times \overrightarrow{C_{n}} \cong B \times \overrightarrow{C_{n}}$ and, for fixed $A$ and $\overrightarrow{C_{n}}$, enumerate digraphs $B$ for which the isomorphism holds. Lovász did some work in this area when he introduced the idea of a zero-divisor, providing conditions on $C$ such that there exist $A$ and $B$, with $A \nsubseteq B$, for which the isomorphism $A \times C \cong B \times C$ holds. Specifically, all directed cycles are zero-divisors. In the search for an answer to our question, we first examine automorphisms of digraphs. Secondly, we look at anti-automorphisms. Together, these special functions lead us to a final theorem involving the factorial of a digraph. We conclude this chapter with a summary of our results and thoughts on extending these results to zero-divisors in general.

\subsection{Zero-divisors}

In [4], Lovász introduced the idea of a zero-divisor. He said that any digraph $C$ for which there exist nonisomorphic digraphs $A$ and $B$ with $A \times C \cong B \times C$ is called a zero-divisor. As you can see in Figure 3.1, the graph $K_{2}$ is a zero-divisor because $A \nsucceq B$ but $A \times K_{2} \cong B \times K_{2}$.
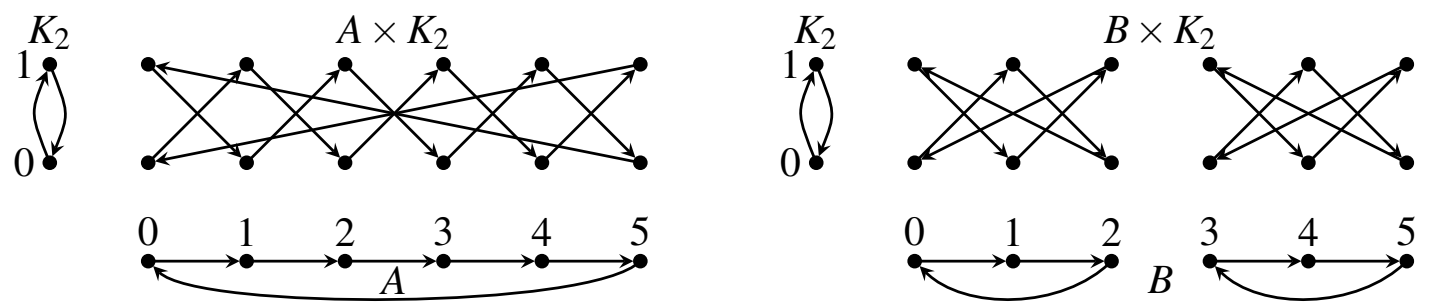

Figure 3.1: $A \times K_{2} \cong B \times K_{2}$, so $K_{2}$ is a zero-divisor 


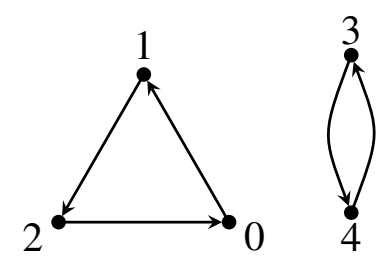

Figure 3.2: A representation of the disjoint union $\overrightarrow{C_{3}}+\overrightarrow{C_{2}}$

Exploring the properties of these zero-divisors, Lovász was able to characterize all zero-divisors with the following theorem.

THEOREM 3.1. (Lovász [4], Theorem 8) A digraph $C$ is a zero-divisor if and only if there is a homomorphism $C \rightarrow \overrightarrow{C_{p_{1}}}+\overrightarrow{C_{p_{2}}}+\cdots+\overrightarrow{C_{p_{k}}}$ for prime numbers $p_{1}, p_{2}, \ldots, p_{k}$. Note that the operation + is the disjoint union of graphs where each graph is drawn separately without any edges connecting vertices of one to vertices of another. The result is the disjoint union. An example can be see in Figure 3.2.

We will focus our attention on connected zero-divisors. Once we have results for these, they can be easily extended to disconnected zero-divisors by applying our result to each component separately. Suppose $C$ is a connected zero-divisor. Not only do we have a homomorphism $C \rightarrow \overrightarrow{C_{p_{1}}}+\overrightarrow{C_{p_{2}}}+\cdots+\overrightarrow{C_{p_{k}}}$, but by Lemma 4.1, the image of $C$ must be in one component of $\overrightarrow{C_{p_{1}}}+\overrightarrow{C_{p_{2}}}+\cdots+\overrightarrow{C_{p_{k}}}$. So we need only consider single directed cycles $\overrightarrow{C_{p}}$ for some prime $p$. As we do, keep in mind the following theorem from Lovász that was proved in Chapter 2.

Theorem 2.4. (Lovász [4], Theorem 6) Let $A, B, C$ and $D$ be digraphs. If $A \times C \cong B \times C$ and there is a homomorphism from $D$ to $C$, then $A \times D \cong B \times D$.

The next corollary follows directly from Theorem 2.4. The proof is trivial but the corollary is stated in a manner that pertains more directly to our purpose. 
COROLlaRY 3.2. If $A \times \overrightarrow{C_{n}} \cong B \times \overrightarrow{C_{n}}$ and there is a homomorphism from $C$ into $\overrightarrow{C_{n}}$, then $A \times C \cong B \times C$.

Now by our Corollary 3.2, any results that we develop for $\overrightarrow{C_{n}}$ can be easily extended to all zero-divisors that have a homomorphism into $\vec{C}_{n}$.

\subsection{Automorphisms}

In our quest to classify all digraphs $B$, dependent upon $A$, such that $A \times C \cong B \times C$ for zero-divisors $C$, we begin by considering a type of permutation on the vertices of $A$, called an automorphism.

DEFINITION 3.3. An automorphism, $\alpha$, on a digraph $A$ is a permutation on the vertices of $A$ with the added requirement that $a a^{\prime}$ is an edge of $A$ if and only if $\alpha(a) \alpha\left(a^{\prime}\right)$ is also an edge of $A$. We denote the collection of all automorphisms on a given digraph $A$ as $\operatorname{Aut}(A)$.

As an example of an automorphism, consider the digraphs $A$ and $\overrightarrow{C_{6}}$ in Figure 3.3. For $\operatorname{digraph} A$, the permutation $\alpha=(23)$ is an automorphism by the following:

$$
\begin{aligned}
& 12 \in E(A) \Longleftrightarrow \alpha(1) \alpha(2)=13 \in E(A) \\
& 13 \in E(A) \Longleftrightarrow \alpha(1) \alpha(3)=12 \in E(A) \\
& 23 \in E(A) \Longleftrightarrow \alpha(2) \alpha(3)=32 \in E(A) \\
& 32 \in E(A) \Longleftrightarrow \alpha(3) \alpha(2)=23 \in E(A)
\end{aligned}
$$

By a similar argument, $(03)(14)(25)$ is an automorphism for $\vec{C}_{6}$.

Given an automorphism $\alpha$ for a $\operatorname{digraph} A$, recall that we can create a new $\operatorname{digraph} A^{\alpha}$ with $V\left(A^{\alpha}\right)=V(A)$ and $E\left(A^{\alpha}\right)=\left\{a \alpha\left(a^{\prime}\right): a a^{\prime} \in E(A)\right\}$. For the digraph $A$ in Figure 3.3, consider the products $A \times K_{2}$ and $A^{(23)} \times K_{2}$ found in Figure 3.4. Observe these two are 
isomorphic, as pointed out in Chapter 1, even though $A \supsetneqq A^{(23)}$. Keeping in mind that (23) is an automorphism of order 2 and $K_{2} \cong \overrightarrow{C_{2}}$, consider the following Proposition 3.4.
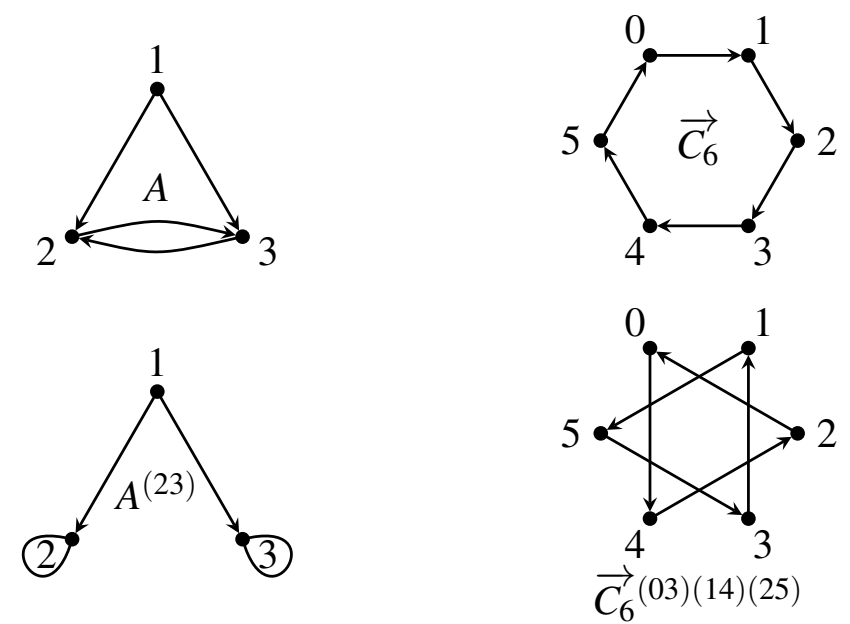

Figure 3.3: Digraph $A$ with automorphism (23) and corresponding power graph. Digraph $\overrightarrow{C_{6}}$ with automorphism $(03)(14)(25)$ and corresponding power graph.
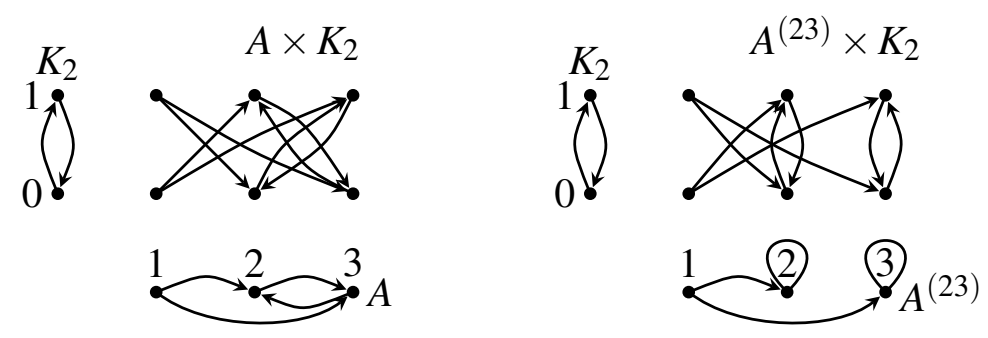

Figure 3.4: A digraph $A$ and automorphism (23) with $A \times K_{2} \cong A^{(23)} \times K_{2}$ 
Proposition 3.4. Suppose $A$ is a digraph and $\phi \in \operatorname{Aut}(A)$ for which $\phi^{k}=i d$. Then

$$
A \times \overrightarrow{C_{k}} \cong A^{\phi} \times \overrightarrow{C_{k}}
$$

Proof. Let $A$ be a digraph and $\phi \in \operatorname{Aut}(A)$ with $\phi^{k}=i d$. In order to prove $A \times \overrightarrow{C_{k}} \cong A^{\phi} \times \overrightarrow{C_{k}}$, we need to provide an isomorphism from the vertices of $A \times \overrightarrow{C_{k}}$ to the vertices of $A^{\phi} \times \overrightarrow{C_{k}}$.

Define $\mu: V\left(A \times \overrightarrow{C_{k}}\right) \rightarrow V\left(A^{\phi} \times \overrightarrow{C_{k}}\right)$ such that

$$
\mu(a, i)=\left(\phi^{i}(a), i\right)
$$

where $\phi^{0}(a)=i d(a)=a$. Because $\phi$ is an automorphism for $A$, we know that $\phi$ is a permutation on the vertices of $A$. So for any vertex $a$ in $A$, we must also have the vertices $\phi^{-1}(a)$ and $\phi^{-i}(a)$ in $V(A)$. And, for any vertex $(a, i)$ in $A^{\phi} \times \overrightarrow{C_{k}}$, there is a vertex $\left(\phi^{-i}(a), i\right)$ in $A \times \overrightarrow{C_{k}}$ such that $\mu\left(\phi^{-i}(a), i\right)=\left(\phi^{i}\left(\phi^{-i}(a)\right), i\right)=(a, i)$. Thus $\mu$ is surjective.

Next suppose there are two distinct vertices $(a, i)$ and $(b, j)$ in $A \times \overrightarrow{C_{k}}$ that map to the same place, $\mu(a, i)=\mu(b, j)$. Then by our definition of $\mu$, we must have $\left(\phi^{i}(a), i\right)=\left(\phi^{j}(b), j\right)$. This is only true if $i=j$ and $\phi^{i}(a)=\phi^{j}(b)$ which together give us $\phi^{i}(a)=\phi^{i}(b)$. And since $\phi$ is a permutation, $a=b$. So $(a, i)=(b, j)$ and $\mu$ is injective.

In order to show that $\mu$ is a homomorphism, consider any edge $(a, i)\left(a^{\prime}, i+1\right)$ in $A \times \overrightarrow{C_{k}}$ and see if it maps to an edge in $A^{\phi} \times \overrightarrow{C_{k}}$. Note if $i=k-1$ then $i+1=0$. Consider the 
following equivalences. (Recall $\phi$ is an automorphism.)

$$
\begin{aligned}
(a, i)\left(a^{\prime}, i+1\right) \in E\left(A \times \overrightarrow{C_{k}}\right) & \Longleftrightarrow a a^{\prime} \in E(A) \\
& \Longleftrightarrow \phi^{i}(a) \phi^{i}\left(a^{\prime}\right) \in E(A) \\
& \Longleftrightarrow \phi^{i}(a) \phi^{i+1}\left(a^{\prime}\right) \in E\left(A^{\phi}\right) \\
& \Longleftrightarrow\left(\phi^{i}(a), i\right)\left(\phi^{i+1}\left(a^{\prime}\right), i+1\right) \in E\left(A^{\phi} \times \overrightarrow{C_{k}}\right) \\
& \Longleftrightarrow \mu(a, i) \mu\left(a^{\prime}, i+1\right) \in E\left(A^{\phi} \times \overrightarrow{C_{k}}\right) .
\end{aligned}
$$

And thus $\mu$ is a homomorphism.

Finally we need to show that $\mu^{-1}$ is also a homomorphism. Consider any edge $(a, i)\left(\phi\left(a^{\prime}\right), i+1\right)$ in $A^{\phi} \times \overrightarrow{C_{k}}$. Then,

$$
\begin{aligned}
(a, i)\left(\phi\left(a^{\prime}\right), i+1\right) \in E\left(A^{\phi} \times \overrightarrow{C_{k}}\right) & \Longleftrightarrow a \phi\left(a^{\prime}\right) \in E\left(A^{\phi}\right) \\
& \Longleftrightarrow a a^{\prime} \in E(A) \\
& \Longleftrightarrow \phi^{-i}(a) \phi^{-i}\left(a^{\prime}\right) \in E(A) \\
& \Longleftrightarrow \phi^{-i}(a) \phi^{-i-1} \phi^{1}\left(a^{\prime}\right) \in E(A) \\
& \Longleftrightarrow \phi^{-i}(a) \phi^{-(i+1)}\left(\phi\left(a^{\prime}\right)\right) \in E(A) \\
& \left.\Longleftrightarrow \phi^{-i}(a), i\right)\left(\phi^{-(i+1)}\left(\phi\left(a^{\prime}\right)\right), i+1\right) \in E\left(A \times \overrightarrow{C_{k}}\right) \\
& \Longleftrightarrow \mu^{-1}(a, i) \mu^{-1}\left(\phi\left(a^{\prime}\right), i+1\right) \in E\left(A \times \overrightarrow{C_{k}}\right)
\end{aligned}
$$

So $\mu^{-1}$ is also a homomorphism, enabling us to conclude that $\mu$ is an isomorphism. Consequently, $A \times \overrightarrow{C_{k}} \cong A^{\phi} \times \overrightarrow{C_{k}}$.

Observe $\overrightarrow{C_{6}}$ and the automorphism (03)(14)(25) provide another illustration of Proposition 3.4 as can be seen in Figure 3.5. 

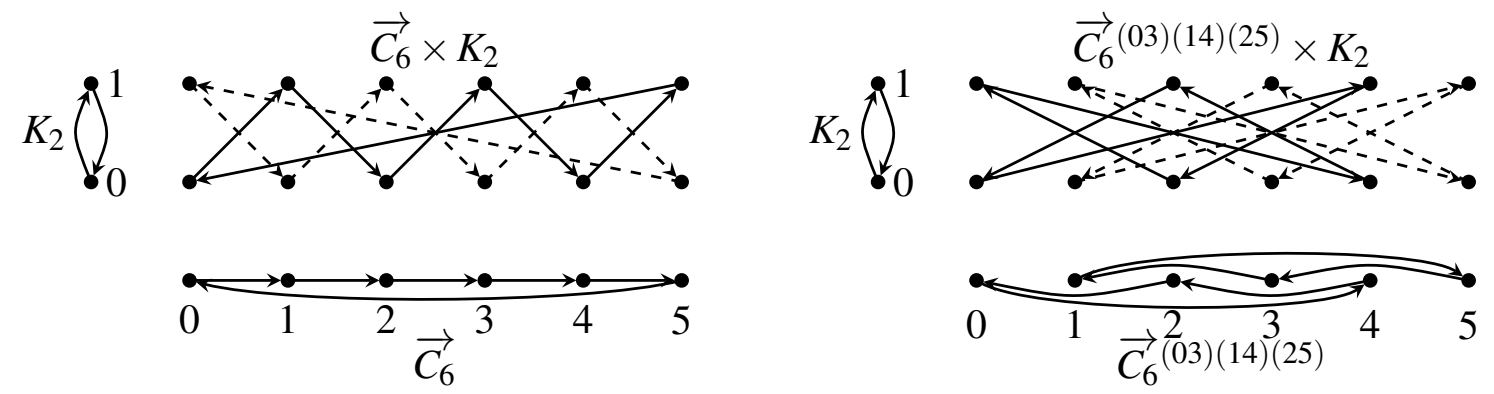

Figure 3.5: An automorphism on $\vec{C}_{6}$ and isomorphic products (Some edges are drawn dashed to emphasize the isomorphism.)

Using Theorem 2.4 mentioned above, we can extend Proposition 3.4 with the following corollary.

Corollary 3.5. Suppose $A$ is a digraph and $\phi \in \operatorname{Aut}(A)$ for which $\phi^{k}=i d$. If $n$ is a multiple of $k$, then

$$
A \times \overrightarrow{C_{n}} \cong A^{\phi} \times{\overrightarrow{C_{n}}}
$$

Proof. Define $\theta: V\left(\overrightarrow{C_{n}}\right) \rightarrow V\left(\overrightarrow{C_{k}}\right)$ by $\theta(i)=i \bmod k$ for any vertex $i \in V\left(\overrightarrow{C_{n}}\right)$ where $i \in$ $\mathbb{Z}_{n}$. Observe for any edge $(i)(i+1) \in \overrightarrow{C_{n}}$, we can express the integers as $i=s k+t$ and consequently $i+1=s k+t+1$ where $t<k$. Then

$$
\theta(i) \theta(i+1)=\theta(s k+t) \theta(s k+t+1)=(t)(t+1)
$$

Note if $t+1=k$, then $\theta(s k+t+1)=(t+1) \bmod k=0$. And finally $(t)(t+1) \in E\left(\overrightarrow{C_{k}}\right)$ by the structure of $\overrightarrow{C_{k}}$. So $\theta$ is a homomorphism from $\overrightarrow{C_{n}}$ to $\overrightarrow{C_{k}}$. Together with Proposition 3.4, the conditions in Theorem 2.4 are fulfilled and thus $A \times \overrightarrow{C_{n}} \cong A^{\phi} \times \overrightarrow{C_{n}}$.

To illustrate a result of this corollary, consider Figure 3.6. Digraphs $A$ and $A^{(23)}$ are 
identical to those in Figure 3.4. Because (23) is an order 2 automorphism and 4 is a multiple of 2, Corollary 3.5 provides us with isomorphic products $A \times \overrightarrow{C_{4}}$ and $A^{(23)} \times \overrightarrow{C_{4}}$. The isomorphism $\psi: V\left(A \times \overrightarrow{C_{4}}\right) \rightarrow V\left(A^{(23)} \times \overrightarrow{C_{4}}\right)$ is defined in a manner similar to the one described in the proof of Proposition 3.4:

$$
\begin{array}{lll}
\psi(1,0)=(1,0) & \psi(2,0)=(2,0) & \psi(3,0)=(3,0) \\
\psi(1,1)=(1,1) & \psi(2,1)=(3,1) & \psi(3,1)=(2,1) \\
\psi(1,2)=(1,2) & \psi(2,2)=(2,2) & \psi(3,2)=(3,2) \\
\psi(1,3)=(1,3) & \psi(2,3)=(3,3) & \psi(3,3)=(2,3)
\end{array}
$$
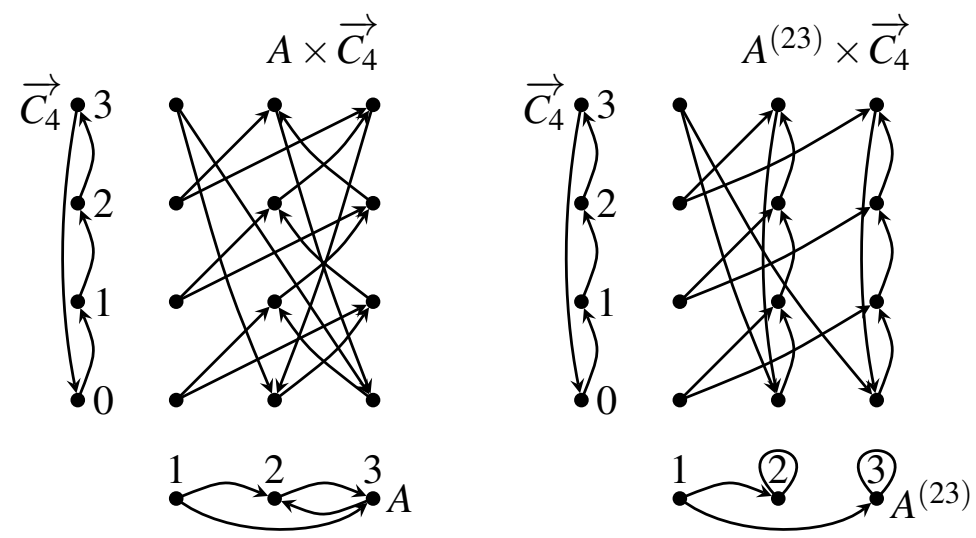

Figure 3.6: A digraph $A$ and automorphism (23) with $A \times \overrightarrow{C_{4}} \cong A^{(23)} \times \overrightarrow{C_{4}}$

\subsection{Anti-automorphisms}

Next we turn to another type of permutation called an anti-automorphism. These were first introduced in [2].

DEFINITION 3.6. A bijection $\mu: V(A) \rightarrow V(A)$ is an anti-automorphism on the digraph $A$ if $\mu$ has the property that $a a^{\prime} \in E(A)$ if and only if $\mu(a) \mu^{-1}\left(a^{\prime}\right) \in E(A)$ for all pairs 
$a, a^{\prime} \in V(A)$. The collection of all anti-automorphisms on $A$ is denoted $\operatorname{Ant}(A)$.

An example of an anti-automorphism on the graph $C_{6}$ is given in Figure 3.7. The permutation $(0341)(25)$ is an anti-automorphism on $C_{6}$ that can be seen as follows. Keep in mind that each edge in the graph is equivalent to two edges in the corresponding digraph. The digraph edges are used to verify that $\alpha=(0341)(25)$ is in fact an anti-automorphism on $C_{6}$.

$$
\begin{array}{lll}
01 \in E\left(C_{6}\right) & \Longleftrightarrow 34 \in E\left(C_{6}\right) & 10 \in E\left(C_{6}\right) \Longleftrightarrow 01 \in E\left(C_{6}\right) \\
12 \in E\left(C_{6}\right) \Longleftrightarrow 05 \in E\left(C_{6}\right) & 21 \in E\left(C_{6}\right) \Longleftrightarrow 54 \in E\left(C_{6}\right) \\
23 \in E\left(C_{6}\right) \Longleftrightarrow 50 \in E\left(C_{6}\right) & 32 \in E\left(C_{6}\right) \Longleftrightarrow 45 \in E\left(C_{6}\right) \\
34 \in E\left(C_{6}\right) \Longleftrightarrow 43 \in E\left(C_{6}\right) & 43 \in E\left(C_{6}\right) \Longleftrightarrow 10 \in E\left(C_{6}\right) \\
45 \in E\left(C_{6}\right) \Longleftrightarrow 12 \in E\left(C_{6}\right) & 54 \in E\left(C_{6}\right) \Longleftrightarrow 23 \in E\left(C_{6}\right) \\
50 \in E\left(C_{6}\right) \Longleftrightarrow 21 \in E\left(C_{6}\right) & 05 \in E\left(C_{6}\right) \Longleftrightarrow 32 \in E\left(C_{6}\right)
\end{array}
$$
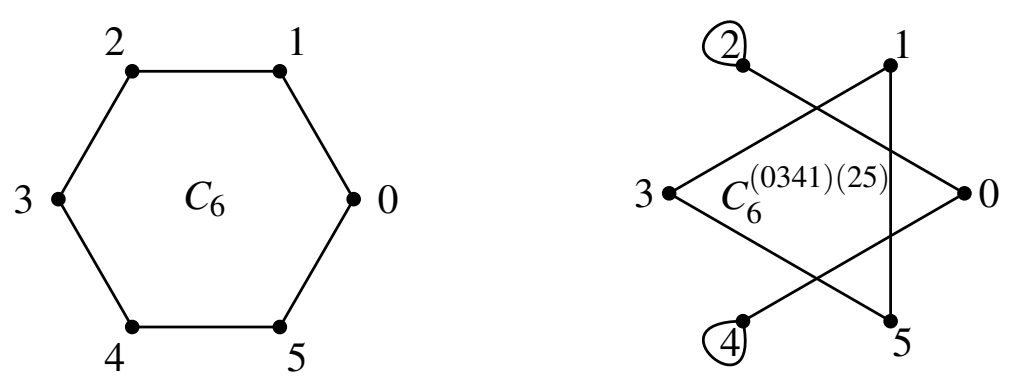

Figure 3.7: An anti-automorphism (0341)(25) on $C_{6}$ and the corresponding power graph $C_{6}^{(0341)(25)}$ (both drawn as graphs)

Recall Lovászs' characterization that a digraph $C$ is a zero-divisor if and only if there is a homomorphism $C \rightarrow \overrightarrow{C_{p_{1}}}+\overrightarrow{C_{p_{2}}}+\cdots+\overrightarrow{C_{p_{k}}}$ for prime $p_{i}$. Also recall that every graph can be expressed as a symmetric digraph. Of the directed cycles $\overrightarrow{C_{p}}$ for a prime $p$, only $\overrightarrow{C_{2}}$ 
is symmetric. So for a graph to be a zero-divisor, there must be a homomorphism into $\overrightarrow{C_{2}}$ which is isomorphic to $K_{2}$.

Focusing our attention on zero-divisors that are graphs, as opposed to digraphs, the following result is specifically for the zero-divisor $K_{2}$. It was first proved for graphs $A$ and $B$ in [2]. Here we generalize to digraphs $A$ and $B$.

Proposition 3.7. For digraphs $A$ and $B, A \times K_{2} \cong B \times K_{2}$ if and only if $B \cong A^{\mu}$ for some $\mu \in \operatorname{Ant}(A)$.

Proof. To begin, suppose $A \times K_{2} \cong B \times K_{2}$. In order to show $B \cong A^{\mu}$ for some $\mu \in \operatorname{Ant}(A)$ we will create an isomorphism $B \rightarrow A^{\mu}$. Because $A \times K_{2} \cong B \times K_{2}$, there is an isomorphism $\psi: V\left(A \times K_{2}\right) \rightarrow V\left(B \times K_{2}\right)$ such that $\psi(a, i)=(\pi(a, i), i)$, for $i \in\{0,1\}$, where $\pi$ is the projection of $\psi(a, i)$ into $B$. Now define functions $\alpha, \beta: V(A) \rightarrow V(B)$ where $\alpha(a)=\pi(a, 0)$ and $\beta(a)=\pi(a, 1)$. Because $\psi$ is an isomorphism, $\alpha$ and $\beta$ must be bijections.

Now consider the composition $\alpha^{-1} \beta: V(A) \rightarrow V(B) \rightarrow V(A)$. Then,

$$
\begin{aligned}
a a^{\prime} \in E(A) & \Longleftrightarrow(a, 0)\left(a^{\prime}, 1\right) \text { and }(a, 1)\left(a^{\prime}, 0\right) \in E\left(A \times K_{2}\right) \\
& \Longleftrightarrow \psi(a, 0) \psi\left(a^{\prime}, 1\right) \text { and } \psi(a, 1) \psi\left(a^{\prime}, 0\right) \in E\left(B \times K_{2}\right) \\
& \Longleftrightarrow(\pi(a, 0), 0)\left(\pi\left(a^{\prime}, 1\right), 1\right) \text { and }(\pi(a, 1), 1)\left(\pi\left(a^{\prime}, 0\right), 0\right) \in E\left(B \times K_{2}\right) \\
& \Longleftrightarrow(\alpha(a), 0)\left(\beta\left(a^{\prime}\right), 1\right) \text { and }(\beta(a), 1)\left(\alpha\left(a^{\prime}\right), 0\right) \in E\left(B \times K_{2}\right) \\
& \Longleftrightarrow \alpha(a) \beta\left(a^{\prime}\right) \text { and } \beta(a) \alpha\left(a^{\prime}\right) \in E(B) .
\end{aligned}
$$

So for any edge $a a^{\prime}$ in $A$, we have edges $\alpha(a) \beta\left(a^{\prime}\right)$ and $\beta(a) \alpha\left(a^{\prime}\right)$ in $B$. Hence for any edge $b b^{\prime}$ in $B$, the edges $\alpha^{-1}(b) \beta^{-1}\left(b^{\prime}\right)$ and $\beta^{-1}(b) \alpha^{-1}\left(b^{\prime}\right)$ are in $A$. 
To conclude that $\alpha^{-1} \beta$ is an anti-automorphism, we know

$$
\begin{aligned}
a a^{\prime} \in E(A) & \Longleftrightarrow \alpha(a) \beta\left(a^{\prime}\right) \text { and } \beta(a) \alpha\left(a^{\prime}\right) \in E(B) \\
& \Longleftrightarrow \beta^{-1} \alpha(a) \alpha^{-1} \beta\left(a^{\prime}\right) \text { and } \alpha^{-1} \beta(a) \beta^{-1} \alpha\left(a^{\prime}\right) \in E(A) \\
& \Longleftrightarrow\left(\alpha^{-1} \beta\right)^{-1}(a)\left(\alpha^{-1} \beta\right)\left(a^{\prime}\right) \text { and }\left(\alpha^{-1} \beta\right)(a)\left(\alpha^{-1} \beta\right)^{-1}\left(a^{\prime}\right) \in E(A)
\end{aligned}
$$

So $\alpha^{-1} \beta$ is in fact an anti-automorphism. Let $\mu=\alpha^{-1} \beta$. Now we show that there is an isomorphism $V\left(A^{\mu}\right) \rightarrow V(B)$.

Recall $\alpha: V(A) \rightarrow V(B)$ so $\alpha: V\left(A^{\mu}\right) \rightarrow V(B)$ since $V(A)=V\left(A^{\mu}\right)$ and $\alpha$ is a bijection. Then for any edge $a a^{\prime}$ in $A, a \mu\left(a^{\prime}\right)=a \alpha^{-1} \beta\left(a^{\prime}\right)$ is in $A^{\mu}$. Then mapping this edge to $B$ using $\alpha$, we see $\alpha(a) \alpha\left(\mu\left(a^{\prime}\right)\right)=\alpha(a) \alpha\left(\alpha^{-1} \beta\left(a^{\prime}\right)\right)=\alpha(a) \beta\left(a^{\prime}\right)$ which is an edge in $B$. So $\alpha$ is a homomorphism.

On the other hand, for any edge $b b^{\prime}$ in $B, \alpha^{-1}(b) \beta^{-1}\left(b^{\prime}\right)$ is an edge in $A$. And thus $\alpha^{-1}(b) \mu\left(\beta^{-1}\left(b^{\prime}\right)\right)=\alpha^{-1}(b) \alpha^{-1}\left(b^{\prime}\right)$ is an edge in $A^{\mu}$. This shows that $\alpha^{-1}$ is a homomorphism. So $\alpha$ is an isomorphism and $B \cong A^{\mu}=A^{\alpha^{-1} \beta}$.

Conversely, for any $\mu \in \operatorname{Ant}(A)$, show that $A \times K_{2} \cong A^{\mu} \times K_{2}$. Define a function $\psi: A \times K_{2} \rightarrow A^{\mu} \times K_{2}$ by $\psi(a, i)=\left(\mu^{i}(a), i\right)$ where $i \in\{0,1\}$ and $\mu^{0}=i d$. Then for any edge $(a, 0)\left(a^{\prime}, 1\right)$ in $A \times K_{2}$, observe

$$
\begin{aligned}
(a, 0)\left(a^{\prime}, 1\right) \in E\left(A \times K_{2}\right) & \Longleftrightarrow\left(a, a^{\prime}\right) \in E(A) \\
& \Longleftrightarrow\left(a, \mu\left(a^{\prime}\right)\right) \in E\left(A^{\mu}\right) \\
& \Longleftrightarrow(a, 0)\left(\mu\left(a^{\prime}\right), 1\right) \in E\left(A^{\mu} \times K_{2}\right) \\
& \Longleftrightarrow \psi(a, 0) \psi\left(a^{\prime}, 1\right) \in E\left(A^{\mu} \times K_{2}\right)
\end{aligned}
$$


Then for any edge $(a, 1)\left(a^{\prime}, 0\right)$ in $A \times K_{2}$, since $\mu$ is an anti-automorphism, observe

$$
\begin{aligned}
(a, 1)\left(a^{\prime}, 0\right) \in E\left(A \times K_{2}\right) & \Longleftrightarrow\left(a, a^{\prime}\right) \in E(A) \\
& \Longleftrightarrow\left(\mu(a), \mu^{-1}\left(a^{\prime}\right)\right) \in E(A) \\
& \Longleftrightarrow\left(\mu(a), a^{\prime}\right) \in E\left(A^{\mu}\right) \\
& \Longleftrightarrow(\mu(a), 1)\left(a^{\prime}, 0\right) \in E\left(A^{\mu} \times K_{2}\right) \\
& \Longleftrightarrow \psi(a, 1) \psi\left(a^{\prime}, 0\right) \in E\left(A^{\mu} \times K_{2}\right)
\end{aligned}
$$

From these two equivalences, $\psi$ is a homomorphism.

On the other hand, for any edge $(a, 0)\left(\mu\left(a^{\prime}\right), 1\right)$ in $A^{\mu} \times K_{2}$, observe

$$
\begin{aligned}
(a, 0)\left(\mu\left(a^{\prime}\right), 1\right) \in E\left(A^{\mu} \times K_{2}\right) & \Longleftrightarrow a \mu\left(a^{\prime}\right) \in E\left(A^{\mu}\right) \\
& \Longleftrightarrow a a^{\prime} \in E(A) \\
& \Longleftrightarrow(a, 0)\left(a^{\prime}, 1\right) \in E\left(A \times K_{2}\right) \\
& \Longleftrightarrow \psi^{-1}(a, 0) \psi^{-1}\left(\mu\left(a^{\prime}\right), 1\right) \in E\left(A \times K_{2}\right)
\end{aligned}
$$

Finally, for any edge $(a, 1)\left(\mu\left(a^{\prime}\right), 0\right)$ in $A^{\mu} \times K_{2}$, observe

$$
\begin{aligned}
(a, 1)\left(\mu\left(a^{\prime}\right), 0\right) \in E\left(A^{\mu} \times K_{2}\right) & \Longleftrightarrow a \mu\left(a^{\prime}\right) \in E\left(A^{\mu}\right) \\
& \Longleftrightarrow a a^{\prime} \in E(A) \\
& \Longleftrightarrow \mu^{-1}(a) \mu\left(a^{\prime}\right) \in E(A) \\
& \Longleftrightarrow\left(\mu^{-1}(a), 1\right)\left(\mu\left(a^{\prime}\right), 0\right) \in E\left(A \times K_{2}\right) \\
& \Longleftrightarrow \psi^{-1}(a, 1) \psi^{-1}\left(\mu\left(a^{\prime}\right), 0\right) \in E\left(A \times K_{2}\right)
\end{aligned}
$$

which comes from the fact that $\mu$ is an anti-automorphism. By the last two equivalences, 
$\psi^{-1}$ is also a homomorphism. And thus $\psi: A \times K_{2} \rightarrow A^{\mu} \times K_{2}$ is an isomorphism implying $A \times K_{2} \cong A^{\mu} \times K_{2}$.

We presented an example of an anti-automorphism on $\vec{C}_{6}$ in Figure 3.7. Using these same graphs, their products with $K_{2}$ can found in Figure 3.8. As emphasized by the solid and dashed lines, the graph products are isomorphic which follows directly from Proposition 3.7.
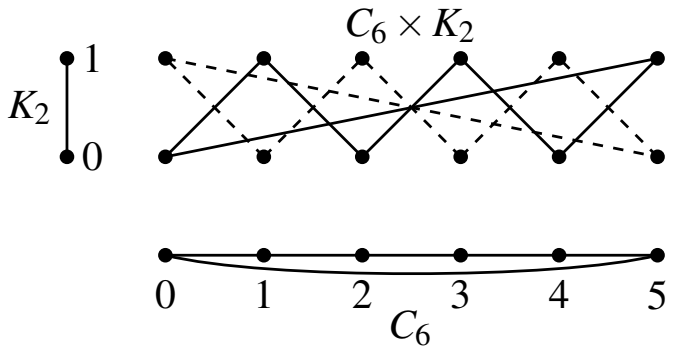
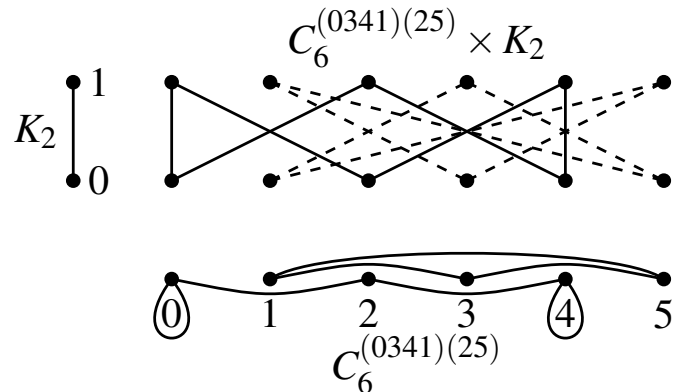

Figure 3.8: An anti-automorphism for $C_{6}$ and isomorphic products

\subsection{Factorial of a digraph}

Remember our goal of finding all $B$, dependent upon $A$, such that $A \times \overrightarrow{C_{p}} \cong B \times \overrightarrow{C_{p}}$. We have results in this direction using automorphisms and anti-automorphisms. In this section, we will generalize the proof for anti-automorphisms as we extend it from the zero-divisor $K_{2}$ to zero-divisors $\overrightarrow{C_{p}}$ for any number $p$. In order to do this, we need to define the factorial of a digraph. This was first developed by Hammack in [2].

Definition 3.8. Given any digraph $A$, the factorial of $A$, denoted $A$ !, is defined as follows:

$$
\begin{gathered}
V(A !)=\{\alpha: \alpha \text { is a permutation on } V(A)\} \\
E(A !)=\left\{\alpha \beta: a a^{\prime} \in E(A) \text { if and only if } \alpha(a) \beta\left(a^{\prime}\right) \in E(A)\right\} .
\end{gathered}
$$




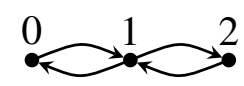

A

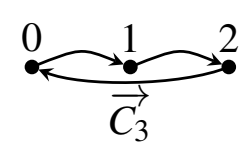

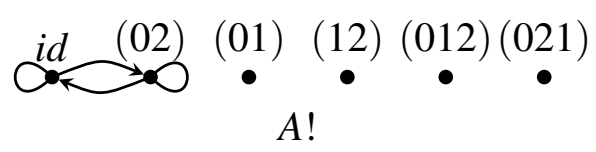

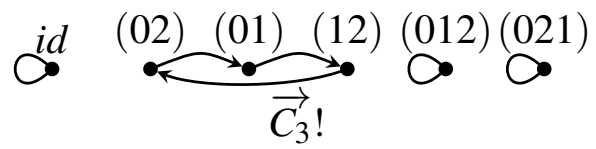

Figure 3.9: Examples of digraphs and their corresponding factorials

For example, consider the digraphs in Figure 3.9 and their corresponding factorials. For $\operatorname{digraph} \overrightarrow{C_{3}}$, let $\alpha=(02)$ and $\beta=(01)$. Observe

$$
\begin{aligned}
& 01 \in E\left(\overrightarrow{C_{3}}\right) \Longleftrightarrow \alpha(0) \beta(1)=20 \in E\left(\overrightarrow{C_{3}}\right) \\
& 12 \in E\left(\overrightarrow{C_{3}}\right) \Longleftrightarrow \alpha(1) \beta(2)=12 \in E\left(\overrightarrow{C_{3}}\right) \\
& 20 \in E\left(\overrightarrow{C_{3}}\right) \Longleftrightarrow \alpha(2) \beta(0)=01 \in E\left(\overrightarrow{C_{3}}\right)
\end{aligned}
$$

resulting in the edge $(\alpha)(\beta)=(02)(01)$ in $\overrightarrow{C_{3}}$ !.

Now consider the following theorem:

THEOREM 3.9. For digraphs $A$ and $B$, and directed cycle $\overrightarrow{C_{p}}$ of length $p, A \times \overrightarrow{C_{p}} \cong B \times \overrightarrow{C_{p}}$ if and only if $B \cong A^{\alpha_{0}}$ where $A$ ! has a closed walk of length $p$ beginning and ending at $\alpha_{0}$, say $\left(\alpha_{0}\right)\left(\alpha_{1}\right)\left(\alpha_{2}\right) \ldots\left(\alpha_{p-1}\right)\left(\alpha_{0}\right)$, such that $\alpha_{0} \alpha_{1} \alpha_{2} \cdots \alpha_{p-1}=i d$.

Before we begin the proof, let us clarify exactly how this theorem extends the previous results. Recall Proposition 3.4 says for any digraph $A$ and $\phi \in \operatorname{Aut}(A)$ for which $\phi^{k}=i d$ then $A \times \overrightarrow{C_{k}} \cong A^{\phi} \times \overrightarrow{C_{k}}$. Now if $\phi$ is an automorphism on $A$, then for all $a a^{\prime}$ in $E(A)$, the edge $\phi(a) \phi\left(a^{\prime}\right)$ is also in $A$. So $(\phi)(\phi)$ is a loop in the factorial. And thus we have a closed walk of length $k$ beginning and ending with $\phi$, namely $(\phi)(\phi) \cdots(\phi)(\phi)$, such that $(\phi)(\phi) \cdots(\phi)=(\phi)^{k}=i d$. So the conditions in Theorem 3.9 have been met, giving us 
$A \times \overrightarrow{C_{k}} \cong A^{\phi} \times \overrightarrow{C_{k}}$ as was proven in Proposition 3.4.

Likewise, recall Theorem 3.7 which states: For digraphs $A$ and $B, A \times K_{2} \cong B \times K_{2}$ if and only if $B \cong A^{\mu}$ for some $\mu \in \operatorname{Ant}(A)$. Because $\mu$ is an anti-automorphism, $a a^{\prime}$ is an edge in $A$ if and only if $\mu(a) \mu^{-1}\left(a^{\prime}\right)$ is an edge in $A$. Thus $(\mu)\left(\mu^{-1}\right)$ is an edge in the factorial. Similarly, any edge can be written $\mu(a) \mu^{-1}\left(a^{\prime}\right)$ given that $a a^{\prime}$ is an edge in $A$. Then the edge $a a^{\prime}$ is equivalent to $\mu^{-1} \mu(a) \mu \mu^{-1}\left(a^{\prime}\right)$, so we also have the edge $\left(\mu^{-1}\right)(\mu)$ in $A$ !. From these two edges in the factorial, we have a walk $(\mu)\left(\mu^{-1}\right)(\mu)$ of length 2 beginning and ending with $\mu$. And clearly $\mu \mu^{-1}=i d$. So $A \times K_{2} \cong B \times K_{2}$ as proved in Theorem 3.7 and as generalized in Theorem 3.9.

With the connections to previous sections, consider the following proof of Theorem 3.9 which is based upon the proof for Theorem 3.7.

Proof. First, suppose $A \times \overrightarrow{C_{p}} \cong B \times \overrightarrow{C_{p}}$. Then there is an isomorphism $\Psi: A \times \overrightarrow{C_{p}} \rightarrow B \times \overrightarrow{C_{p}}$ such that, by Theorem 2.5 from Lovász, $\Psi(a, i)=\left(\beta_{i}(a), i\right)$ where $\beta_{i}$ is the projection of $\Psi$ onto $B$. Now consider $\beta_{i}: V(A) \rightarrow V(B)$. Because $\Psi: A \times \overrightarrow{C_{p}} \rightarrow B \times \overrightarrow{C_{p}}$ is an isomorphism, the cardinalities $\left|V\left(A \times \overrightarrow{C_{p}}\right)\right|$ and $\left|V\left(B \times \overrightarrow{C_{p}}\right)\right|$ are equal and thus $|V(A)|=|V(B)|$. Now suppose there exist two vertices in $A$, say $a$ and $a^{\prime}$, such that $\beta_{i}(a)=\beta_{i}\left(a^{\prime}\right)$. Then

$$
\begin{aligned}
\beta_{i}(a)=\beta_{i}\left(a^{\prime}\right) & \Longleftrightarrow\left(\beta_{i}(a), i\right)=\left(\beta_{i}\left(a^{\prime}\right), i\right) \\
& \Longleftrightarrow \Psi(a, i)=\Psi\left(a^{\prime}, i\right) \\
& \Longleftrightarrow(a, i)=\left(a^{\prime}, i\right) \\
& \Longleftrightarrow a=a^{\prime}
\end{aligned}
$$

So $\beta_{i}$ is injective. And together with the fact that $|V(A)|=|V(B)|$, we may conclude that $\beta_{i}: V(A) \rightarrow V(B)$ is a bijection for all $i \in\{0,1, \ldots, p-1\}$. 
Now consider $\beta_{i}^{-1} \beta_{i+1}: V(A) \rightarrow V(B) \rightarrow V(A)$. Each of these is a bijection from $V(A)$ to $V(A)$ and thus a permutation on $V(A)$. Consequently we have vertices $\beta_{i}^{-1} \beta_{i+1}$ in the digraph $A$ !.

Now observe, for any $a a^{\prime} \in E(A)$ :

$$
\begin{aligned}
a a^{\prime} \in E(A) & \Longleftrightarrow(a, i)\left(a^{\prime}, i+1\right) \in E\left(A \times \overrightarrow{C_{p}}\right) \\
& \Longleftrightarrow \Psi(a, i) \Psi\left(a^{\prime}, i+1\right) \in E\left(B \times \overrightarrow{C_{p}}\right) \\
& \Longleftrightarrow\left(\beta_{i}(a), i\right)\left(\beta_{i+1}\left(a^{\prime}\right), i+1\right) \in E\left(B \times \overrightarrow{C_{p}}\right) \\
& \Longleftrightarrow \beta_{i}(a) \beta_{i+1}\left(a^{\prime}\right) \in E(B)
\end{aligned}
$$

Similarly, for any edge $b b^{\prime} \in E(B)$ :

$$
\begin{aligned}
b b^{\prime} \in E(B) & \Longleftrightarrow(b, j)\left(b^{\prime}, j+1\right) \in E\left(B \times \overrightarrow{C_{p}}\right) \\
& \Longleftrightarrow \Psi^{-1}(b, j) \Psi^{-1}\left(b^{\prime}, j+1\right) \in E\left(A \times \overrightarrow{C_{p}}\right) \\
& \Longleftrightarrow\left(\beta_{j}^{-1}(b), j\right)\left(\beta_{j+1}^{-1}\left(b^{\prime}\right), j+1\right) \in E\left(A \times \overrightarrow{C_{p}}\right) \\
& \Longleftrightarrow \beta_{j}^{-1}(b) \beta_{j+1}^{-1}\left(b^{\prime}\right) \in E(A) .
\end{aligned}
$$

Using the above Equivalence 3.1 from edges in $A$ to edges in $B$, we find that for any $a a^{\prime} \in E(A)$ there is a corresponding edge $\beta_{i}(a) \beta_{i+1}\left(a^{\prime}\right)$ in $B$. And from this edge, by Equivalence 3.2, we can map back to the edge $\beta_{i-1}^{-1} \beta_{i}(a) \beta_{i}^{-1} \beta_{i+1}\left(a^{\prime}\right)$ in $A$. By the definition of edges in $A$ !, we now have $\left(\beta_{i-1}^{-1} \beta_{i}\right)\left(\beta_{i}^{-1} \beta_{i+1}\right)$ is in fact an edge in $A$ !. This is true for any $i \in\{0,1, \ldots, p-1\}$. So we have the walk $\left(\beta_{0}^{-1} \beta_{1}\right)\left(\beta_{1}^{-1} \beta_{2}\right)\left(\beta_{2}^{-1} \beta_{3}\right) \cdots\left(\beta_{p-1}^{-1} \beta_{0}\right)\left(\beta_{0}^{-1} \beta_{1}\right)$ in $A$ !. Also notice that $\beta_{0}^{-1} \beta_{1} \beta_{1}^{-1} \beta_{2} \beta_{2}^{-1} \beta_{3} \cdots \beta_{p-1}^{-1} \beta_{0}=\beta_{0}^{-1} \beta_{0}=i d$.

Thus $\beta_{0}^{-1} \beta_{1}$ meets the criteria in the theorem. Now we just need to provide an isomorphism $A^{\beta_{0}^{-1} \beta_{1}} \rightarrow B$. Consider the map $\beta_{0}$. Recall $V(A)=V\left(A^{\beta_{0}^{-1} \beta_{1}}\right)$. The function $\beta_{0}$ is a bijection as noted above, and $\beta_{0}: V\left(A^{\beta_{0}^{-1} \beta_{1}}\right) \rightarrow V(B)$.

Observe, for any edge $a a^{\prime}$ in $A, a \beta_{0}^{-1} \beta_{1}\left(a^{\prime}\right)$ is an edge in $A^{\beta_{0}^{-1} \beta_{1}}$. Then, taking $\beta_{0}$ of both 
vertices, we see $\beta_{0}(a) \beta_{0} \beta_{0}^{-1} \beta_{1}\left(a^{\prime}\right)=\beta_{0}(a) \beta_{1}\left(a^{\prime}\right)$ which is an edge in $B$ by Equivalence 3.1. So $\beta_{0}$ is a homomorphism.

On the other hand, for any edge $b b^{\prime}$ in $B$, by Equivalence 3.2, we have the edge $\beta_{0}^{-1}(b) \beta_{1}^{-1}\left(b^{\prime}\right)$ in $A$. This subsequently gives us the edge $\beta_{0}^{-1}(b) \beta_{0}^{-1} \beta_{1} \beta_{1}^{-1}\left(b^{\prime}\right)$ in $A^{\beta_{0}^{-1} \beta_{1}}$ which equals $\beta_{0}^{-1}(b) \beta_{0}^{-1}\left(b^{\prime}\right)$. So $\beta_{0}^{-1}$ is also a homomorphism. Thus $\beta_{0}$ is an isomorphism from $A^{\beta_{0}^{-1} \beta_{1}}$ to $B$. So we conclude $A^{\beta_{0}^{-1} \beta_{1}} \cong B$.

Conversely, consider a digraph $A$ with $\alpha_{0}$ a permutation on $V(A)$ such that there is a closed walk $\left(\alpha_{0}\right)\left(\alpha_{1}\right)\left(\alpha_{2}\right) \cdots\left(\alpha_{p-1}\right)\left(\alpha_{0}\right)$ in $A$ !, and $\alpha_{0} \alpha_{1} \alpha_{2} \cdots \alpha_{p-1}=i d$. We want to show that $A \times \overrightarrow{C_{p}} \cong A^{\alpha_{0}} \times \overrightarrow{C_{p}}$. In order to do this, define the function $\psi: A \times \overrightarrow{C_{p}} \rightarrow A^{\alpha_{0}} \times \overrightarrow{C_{p}}$ where

$$
\psi((a, i))=\left(\alpha_{0} \alpha_{1} \cdots \alpha_{i}(a), i\right)
$$

Because each $\alpha_{i}$ is a permutation on the vertices of $A$, the composition $\alpha_{0} \alpha_{1} \cdots \alpha_{i}$ of permutations is itself a permutation on the vertices of $A$. So, $\psi$ must be a bijection.

Recall $a a^{\prime} \in E(A)$ and $\left(\alpha_{0}\right)\left(\alpha_{1}\right)\left(\alpha_{2}\right) \cdots\left(\alpha_{p-1}\right)\left(\alpha_{0}\right)$ is a walk in $A$ !. So for any edge $a a^{\prime}$ in $A$, we have the following statements:

$$
\begin{aligned}
a a^{\prime} \in E(A) & \Longleftrightarrow \alpha_{i}(a) \alpha_{i+1}\left(a^{\prime}\right) \in E(A) \\
& \Longleftrightarrow \alpha_{i-1} \alpha_{i}(a) \alpha_{i} \alpha_{i+1}\left(a^{\prime}\right) \in E(A) \\
& \vdots \\
& \Longleftrightarrow \alpha_{0} \alpha_{1} \cdots \alpha_{i-1} \alpha_{i}(a) \alpha_{1} \alpha_{2} \cdots \alpha_{i} \alpha_{i+1}\left(a^{\prime}\right) \in E(A) \\
& \Longleftrightarrow \alpha_{0} \alpha_{1} \cdots \alpha_{i-1} \alpha_{i}(a) \alpha_{0} \alpha_{1} \alpha_{2} \cdots \alpha_{i} \alpha_{i+1}\left(a^{\prime}\right) \in E\left(A^{\alpha_{0}}\right)
\end{aligned}
$$


Keeping in mind $\left(\alpha_{p-1}\right)\left(\alpha_{0}\right)$ is an edge in $A$ ! and $\alpha_{0} \alpha_{1} \alpha_{2} \cdots \alpha_{p-1}=i d$, note that if $i=p-1$ :

$$
\begin{aligned}
a a^{\prime} \in E(A) & \Longleftrightarrow \alpha_{p-1}(a) \alpha_{0}\left(a^{\prime}\right) \in E(A) \\
& \Longleftrightarrow \alpha_{p-2} \alpha_{p-1}(a) \alpha_{p-1} \alpha_{0}\left(a^{\prime}\right) \in E(A) \\
& \vdots \\
& \Longleftrightarrow \alpha_{0} \alpha_{1} \cdots \alpha_{p-1}(a) \alpha_{1} \alpha_{2} \cdots \alpha_{p-1} \alpha_{0}\left(a^{\prime}\right) \in E(A) \\
& \Longleftrightarrow \alpha_{0} \alpha_{1} \cdots \alpha_{p-1}(a) \alpha_{0} \alpha_{1} \alpha_{2} \cdots \alpha_{p-1} \alpha_{0}\left(a^{\prime}\right) \in E\left(A^{\alpha_{0}}\right) \\
& \Longleftrightarrow \alpha_{0} \alpha_{1} \cdots \alpha_{p-1}(a) \alpha_{0}\left(a^{\prime}\right) \in E\left(A^{\alpha_{0}}\right)
\end{aligned}
$$

Then for any edge $(a, i)\left(a^{\prime}, i+1\right), i \in\{0,1, \ldots, p-2\}$, in $A \times \overrightarrow{C_{p}}$, observe

$$
\psi(a, i) \psi\left(a^{\prime}, i+1\right)=\left(\alpha_{0} \alpha_{1} \cdots \alpha_{i-1} \alpha_{i}(a), i\right)\left(\alpha_{0} \alpha_{1} \alpha_{2} \cdots \alpha_{i} \alpha_{i+1}\left(a^{\prime}\right), i+1\right)
$$

is an edge of $A^{\alpha_{0}} \times \overrightarrow{C_{p}}$. Again, if $i=p-1$, for any edge $(a, p-1)\left(a^{\prime}, 0\right)$, we have the edge $\psi(a, p-1) \psi\left(a^{\prime}, 0\right)=\left(\alpha_{0} \alpha_{1} \cdots \alpha_{p-1}(a), p-1\right)\left(\alpha_{0}\left(a^{\prime}\right), 0\right)$ in $A^{\alpha_{0}} \times \overrightarrow{C_{p}}$. So $\psi$ is a homomorphism.

Finally, for any edge $(a, i)\left(\alpha_{0}\left(a^{\prime}\right), i+1\right)$ in $A^{\alpha_{0}} \times \overrightarrow{C_{p}}$, where $i \in\{0,1,2, \ldots, p-2\}$, observe:

$$
\begin{aligned}
& (a, i)\left(\alpha_{0}\left(a^{\prime}\right), i+1\right) \in E\left(A^{\alpha_{0}} \times \overrightarrow{C_{p}}\right) \\
\Longleftrightarrow & a \alpha_{0}\left(a^{\prime}\right) \in E\left(A^{\alpha_{0}}\right) \\
\Longleftrightarrow & \left(\alpha_{0} \alpha_{1} \cdots \alpha_{i}\right)^{-1}(a)\left(\alpha_{0} \alpha_{1} \cdots \alpha_{i+1}\right)^{-1}\left(\alpha_{0}\left(a^{\prime}\right)\right) \in E(A) \\
\Longleftrightarrow & \left(\left(\alpha_{0} \alpha_{1} \cdots \alpha_{i}\right)^{-1}(a), i\right)\left(\left(\alpha_{0} \alpha_{1} \cdots \alpha_{i+1}\right)^{-1}\left(\alpha_{0}\left(a^{\prime}\right)\right), i+1\right) \in E\left(A \times \overrightarrow{C_{p}}\right) \\
\Longleftrightarrow & \psi^{-1}(a, i) \psi^{-1}\left(\alpha_{0}\left(a^{\prime}\right), i+1\right) \in E\left(A \times \overrightarrow{C_{p}}\right)
\end{aligned}
$$


Then similarly if $i=p-1$ :

$$
\begin{aligned}
& (a, p-1)\left(\alpha_{0}\left(a^{\prime}\right), 0\right) \in A^{\alpha_{0}} \times \overrightarrow{C_{p}} \\
\Longleftrightarrow & a \alpha_{0}\left(a^{\prime}\right) \in E\left(A^{\alpha_{0}}\right) \\
\Longleftrightarrow & \left(\alpha_{0} \alpha_{1} \cdots \alpha_{p-1}\right)^{-1}(a) \alpha_{0}^{-1}\left(\alpha_{0}\left(a^{\prime}\right)\right) \in E(A) \\
\Longleftrightarrow & \left(\left(\alpha_{0} \alpha_{1} \cdots \alpha_{p-1}\right)^{-1}(a), p-1\right)\left(\alpha_{0}^{-1}\left(\alpha_{0}\left(a^{\prime}\right)\right), 0\right) \in E\left(A \times \overrightarrow{C_{p}}\right) \\
\Longleftrightarrow & \psi^{-1}(a, p-1) \psi^{-1}\left(\alpha_{0}\left(a^{\prime}\right), 0\right) \in E\left(A \times \overrightarrow{C_{p}}\right)
\end{aligned}
$$

Thus any edge $(a, i)\left(\alpha_{0}\left(a^{\prime}\right), i+1\right)$ in $A^{\alpha_{0}} \times \overrightarrow{C_{p}}$ gives us the edge $\psi^{-1}(a, i) \psi^{-1}\left(\alpha_{0}\left(a^{\prime}\right), i+1\right)$ in $A \times \overrightarrow{C_{p}}$. So $\psi^{-1}$ is also a homomorphism. Thus $\psi$ is an isomorphism, and consequently $A \times \overrightarrow{C_{p}} \cong A^{\alpha_{0}} \times \overrightarrow{C_{p}}$.

To illustrate the results of this theorem, consider the directed cycle $\overrightarrow{C_{3}}$ whose factorial is given in Figure 3.9. There is a closed walk $(02)(01)(12)(02)$ of length 3 in the factorial, but $(02)(01)(12) \neq i d$. So, by Theorem 3.9, $\overrightarrow{C_{3}} \times \overrightarrow{C_{3}} ¥ \overrightarrow{C_{3}}(02) \times \overrightarrow{C_{3}}$. But, $(02)(01)(12)(02)(01)(12)(02)$ is a closed walk of length 6 in the factorial with the composition $(02)(01)(12)(02)(01)(12)$ equaling the identity. So Theorem 3.9 does guarantee the isomorphism $\overrightarrow{C_{3}} \times \overrightarrow{C_{6}} \cong \overrightarrow{C_{3}}(02) \times \overrightarrow{C_{6}}$ as can be seen in Figure 3.10. Again line style is present merely to emphasize the isomorphism.

As a second example, consider the digraph $\overrightarrow{C_{6}}$. Within the factorial, we have the following closed walk of length 6 :

$$
(0124)(1235)(2340)(3451)(4502)(5013)(0124)
$$

With this one, the composition (0124)(1235)(2340)(3451)(4502)(5013) does in fact give 
us the identity. So, Theorem 3.9 ensures the isomorphism $\overrightarrow{C_{6}} \times \overrightarrow{C_{6}} \cong{\overrightarrow{C_{6}}}^{(0124)} \times \overrightarrow{C_{6}}$ as shown in Figure 3.11. As the different style lines emphasize, each product consists of 6 copies of $\vec{C}_{6}$.
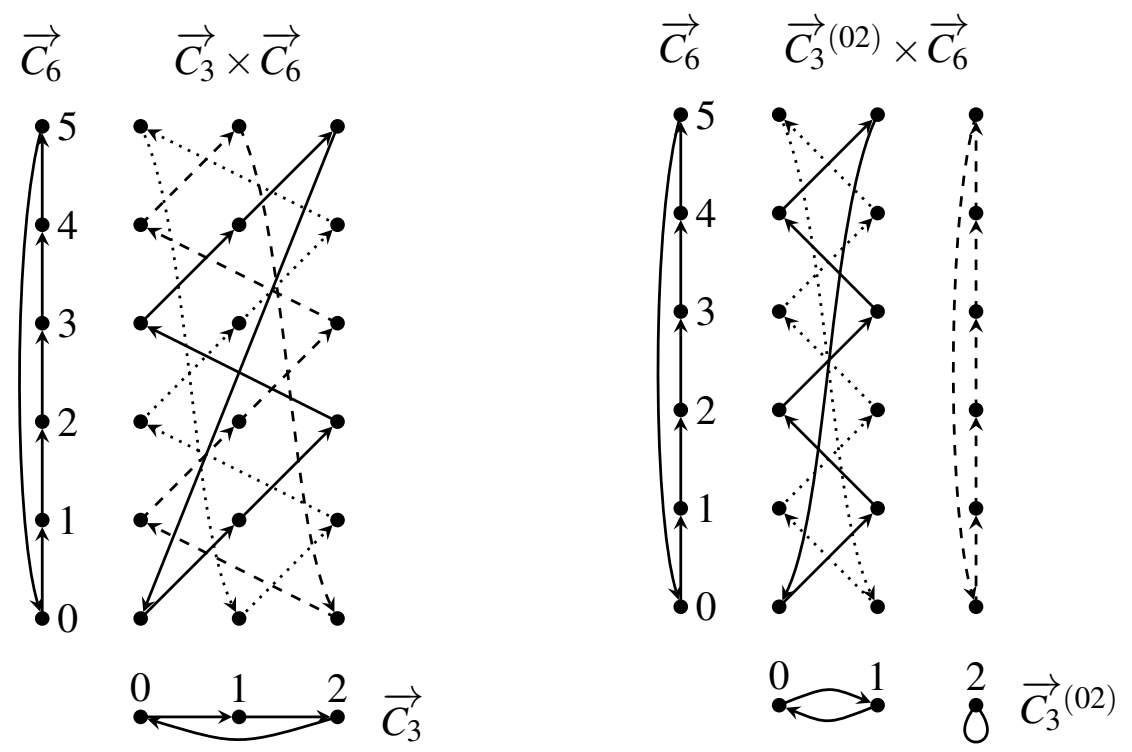

Figure 3.10: Isomorphic products guaranteed by Theorem 3.9

\subsection{Future directions}

Given any zero-divisor of the form $\overrightarrow{C_{n}}$ and any digraph $A$, Theorem 3.9 ennumerates all digraphs $B$ for which $A \times \overrightarrow{C_{n}} \cong B \times \overrightarrow{C_{n}}$. The ultimate goal is to develop a theorem similar to Theorem 3.9 that will hold for all zero-divisors. Let's look specifically at those that are connected. Once a theorem is developed for connected zero-divisors, it can be easily extended to disconnected ones by looking at the components individually. As a step in this direction, recall Lovászs' characterization of zero-divisors, and more specifically connected zero-divisors as developed from Lemma 4.1: A connected digraph $C$ is a zero-divisor if and only if there is a homomorphism $C \rightarrow \overrightarrow{C_{p}}$ for some prime $p$. 

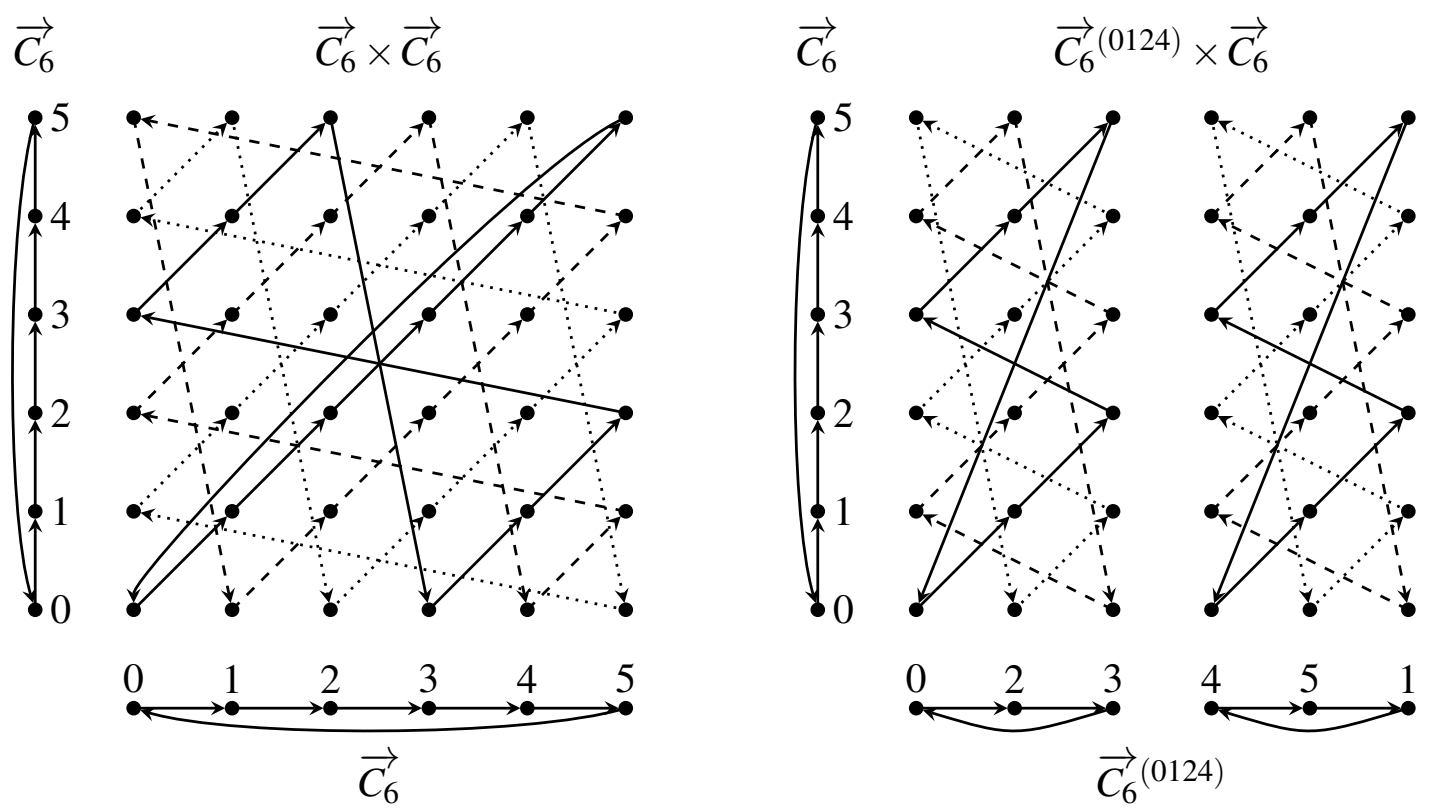

Figure 3.11: Another pair of isomorphic products guaranteed by Theorem 3.9

We already have a result, Theorem 2.6, for zero-divisors with a homomorphism into $\overrightarrow{K_{2}}$. This theorem, first proven in [5], can be found with a proof in chapter 2. Note that these zero-divisors would not have any directed cycles of length 2 or greater.

Suppose $C$ is a zero-divisor with at least one cycle. Let that cycle have length $n$. By Lemma 4.2, there is a homomorphism $\overrightarrow{C_{n}} \rightarrow \overrightarrow{C_{m_{i}}}$ if and if $n$ is a multiple of $m_{i}$. So there are finitely many possible integers $m_{i}$ for which a homomorphism $\overrightarrow{C_{n}} \rightarrow \overrightarrow{C_{m_{i}}}$ exists. And the fact that $\psi: C \rightarrow \overrightarrow{{C_{m}}_{i}}$ is a homomorphism implies that $\left.\psi\right|_{\overrightarrow{C_{n}}}$ is a homomorphism because $\overrightarrow{C_{n}}$ is a subgraph of $C$. So there are finitely many integers $m_{i}$ for which there is a homomorphism $C \rightarrow \overrightarrow{C_{m_{i}}}$. Find the largest value $m$ for which there is a homomorphism $C \rightarrow \overrightarrow{C_{m}}$. (Note that the value $m$ will be the greatest common divisor of the integers $m_{i}$.) Then by Theorem 3.9 we can find all the digraphs $B$ for which $A \times \overrightarrow{C_{m}} \cong B \times \overrightarrow{C_{m}}$. By Theorem 2.4, these same digraphs $B$ will result in $A \times C \cong B \times C$. 
Now consider the size of every cycle in $C$. If there is a homomorphism into $\overrightarrow{C_{m}}$ then every cycle in $C$ must have length which is a multiple of $m$ by Corollary 4.3. So the cycles in $C$ have lengths $m k_{1}, m k_{2}, \ldots, m k_{n}$ where each $k_{i}$ is an integer greater than or equal to 1 . From these cycle lengths, we have homomorphisms $\overrightarrow{C_{m k_{i}}} \rightarrow C$ for each $i$ by mapping the vertices of $\overrightarrow{C_{m k_{i}}}$ directly to the vertices of the cycle in $C$ of length $m k_{i}$. So for every $B$ that satisfies $A \times C \cong B \times C$, those same digraphs $B$ will satisfy $A \times \overrightarrow{C_{m k_{i}}} \cong B \times \overrightarrow{C_{m k_{i}}}$ for every $i$. To summarize, consider the relation of sets.

$$
\begin{gathered}
\bigcap_{i=1}^{n} \Gamma=\bigcap_{i=1}^{n}\left\{A^{\alpha}: A \times \overrightarrow{C_{m k_{i}}} \cong A^{\alpha} \times \overrightarrow{C_{m k_{i}}}\right\} \\
\cup \mid \\
\Lambda=\left\{A^{\alpha}: A \times C \cong A^{\alpha} \times C\right\} \\
\cup \mid \\
\Upsilon=\left\{A^{\alpha}: A \times \overrightarrow{C_{m}} \cong A^{\alpha} \times \overrightarrow{C_{m}}\right\}
\end{gathered}
$$

The goal is to explicitly define $\Lambda$ by determining all $A^{\alpha}$ such that $A \times C \cong A^{\alpha} \times C$. Right now this set is sandwiched between two known sets. Now if some $k_{i}$ is 1 , meaning there is a cycle of length $m$ in $C$, then $\bigcap_{i=1}^{n} \Gamma=\Upsilon$, so we know $\Lambda$. But if there is not a cycle of length $m$ in $C$, the intersection $\bigcap_{i=1}^{n} \Gamma$ will likely have more digraphs than $\Upsilon$, leaving a gap for the exact set $\Lambda$. The refining of these bounds remains an open problem. 


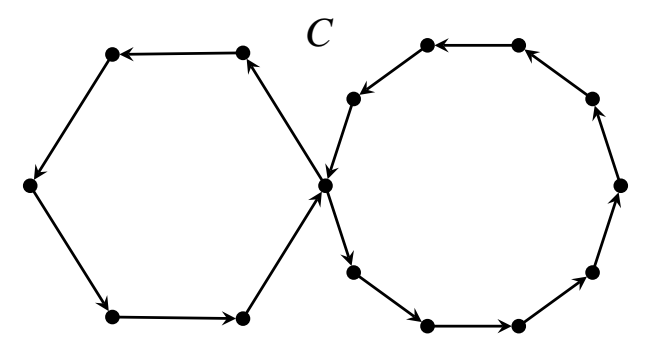

Figure 3.12: A zero-divisor $C$ with homomorphisms $\overrightarrow{C_{6}}, \overrightarrow{C_{10}}, \overrightarrow{C_{16}} \rightarrow C \rightarrow \overrightarrow{C_{2}}$

For example, consider the zero-divisor in Figure 3.12. The cycles in $C$ have lengths 6 , 10, and 16. The largest $m$ for which there is a homomorphism $C \rightarrow \overrightarrow{C_{m}}$ is 2 . There is not a cycle of length 2 , so $\bigcap_{i=1}^{n} \Gamma \supsetneqq \Upsilon$. Examining the factorials and difference between the sets may prove useful. 


\section{Auxiliary Results}

\subsection{Additional results for zero-divisors}

As mentioned in Chapter 3, below are some additional results proven during the work of this thesis. We begin with some results for cycles and zero-divisors in general.

Lemma 4.1. For digraphs $C$ and $G$, if $C$ is connected and there is a homomorphism $\phi: V(C) \rightarrow V(G)$ then $\phi(V(C))$ lies in one component of $G$.

Proof. Let $C$ be connected and let $\phi: V(C) \rightarrow V(G)$ be a homomorphism. Because $C$ is connected, there is a path (with direction unimportant) between every pair of vertices. Since $\phi$ is a homomorphism, every edge in $C$ maps to an edge in $G$. So for any two vertices in $C$, the edges forming the path between them must map to edges in $G$, forming a corresponding path in $G$. Because this is true for every pair of vertices, the image of $C$ is connected. And thus $\phi(V(C))$ lies in one component of $G$.

Lemma 4.2. There is a homomorphism $\psi: \overrightarrow{C_{m}} \rightarrow \overrightarrow{C_{p}}$ if and only if $m$ is a multiple of $p$.

Proof. First, let $\psi: \overrightarrow{C_{m}} \rightarrow \overrightarrow{C_{p}}$ be a homomorphism. Label the vertices in $\overrightarrow{C_{m}}$ as $v_{0}, v_{1}, \ldots, v_{m-1}$ and the vertices in $\overrightarrow{C_{p}}$ as $u_{0}, u_{1}, \ldots, u_{p-1}$. Because of the symmetry of the graphs, we can assume that $\psi\left(v_{i}\right)=u_{i} \bmod p$ for all $i \in\{0,1, \ldots, m-1\}$ without loss of generality. Now because $\psi$ is a homomorphism, the edge $v_{m-1} v_{0}$ in $\overrightarrow{C_{m}}$ maps to a corresponding edge $\psi\left(v_{m-1}\right) \psi\left(v_{0}\right)=u_{(m-1) \bmod p} u_{0} \bmod p$ in $\overrightarrow{C_{p}}$. We know that $u_{(m-1) \bmod p} u_{m} \bmod p$ must be an edge in $\overrightarrow{C_{p}}$. Because the out degree of every vertex in a cycle is exactly $1, u_{0} \bmod p=u_{m} \bmod p$ so $0 \bmod p=m \bmod p$ and thus $m$ must be a multiple of $p$. 
Conversely, assume that $m$ is a multiple of $p$. Define a function $\psi: \overrightarrow{C_{m}} \rightarrow \overrightarrow{C_{p}}$ so that $\psi\left(v_{i}\right)=u_{i} \bmod p$. Thus $\psi$ is a well defined function. Now for any $i \in\{0,1, \ldots, m-2\}$, the edge $v_{i} v_{i+1} \operatorname{maps}$ to $u_{i \bmod p} u_{(i+1) \bmod p}$ which is an edge in $\overrightarrow{C_{p}}$. Finally, consider the edge $v_{m-1} v_{0}$ in $\overrightarrow{C_{m}}$ which maps to $u_{(m-1) \bmod p} u_{0} \bmod p$. Because $m$ is a multiple of $p$, then $m \bmod p=0 \bmod p$. So $u_{(m-1)} \bmod p u_{0 \bmod p}=u_{(m-1) \bmod p} u_{m} \bmod p$ which is clearly an edge in $\overrightarrow{C_{p}}$. So $\psi: \overrightarrow{C_{m}} \rightarrow \overrightarrow{C_{p}}$ is a homomorphism.

COROllary 4.3 . Let $C$ be a zero-divisor with at least one cycle. If $\psi: C \rightarrow \overrightarrow{C_{p}}$ is a homomorphism then every cycle in $C$ has a length which is a multiple of $p$.

Proof. Suppose $\psi: C \rightarrow \overrightarrow{C_{p}}$ is a homomorphism. Then for any cycle $S$ in $C$, the restriction $\left.\psi\right|_{S}$ is a homomorphism into $\overrightarrow{C_{p}}$. Then by Lemma 4.2, we know that the length of $S$ is a multiple of $p$. Because the cycle $S$ in $C$ was arbitrary, this holds for the length of any cycle in $C$.

\subsection{Additional results for power graphs $A^{\alpha}$}

Because the power graphs $A^{\alpha}$ played such a large part in this thesis, below are a few additional results for power graphs where $\alpha$ is an anti-automorphism.

LEMMA 4.4. For any $i, A^{\alpha^{i}} \cong A^{\alpha^{i+2}}$.

Proof. Recall $\alpha: V(A) \rightarrow V(A)$ is a bijection such that $a a^{\prime}$ is an edge in $A$ if and only if $\alpha(a) \alpha^{-1}\left(a^{\prime}\right)$ is an edge in $A$. Because $V\left(A^{\alpha^{i}}\right)=V(A)$ for any $i$, consider the bijection 
$\alpha: V\left(A^{\alpha^{i}}\right) \rightarrow V\left(A^{\alpha^{i+2}}\right)$. Now for any edge $a \alpha^{i}\left(a^{\prime}\right)$ in $A^{\alpha^{i}}$, observe

$$
\begin{aligned}
a \alpha^{i}\left(a^{\prime}\right) \in E\left(A^{\alpha^{i}}\right) & \Longleftrightarrow a a^{\prime} \in E(A) \\
& \Longleftrightarrow \alpha^{1}(a) \alpha^{-1}\left(a^{\prime}\right) \in E(A) \\
& \Longleftrightarrow \alpha(a) \alpha^{i+1}\left(a^{\prime}\right) \in E\left(A^{\alpha^{i+2}}\right) \\
& \Longleftrightarrow \alpha(a) \alpha\left(\alpha^{i}\left(a^{\prime}\right)\right) \in E\left(A^{\alpha^{i+2}}\right)
\end{aligned}
$$

because $\alpha$ is an anti-automorphism of $A$. So $\alpha: V\left(A^{\alpha^{i}}\right) \rightarrow V\left(A^{\alpha^{i+2}}\right)$ is a homomorphism.

On the other hand, for any edge $a \alpha^{i+2}\left(a^{\prime}\right)$ in $A^{\alpha^{i+2}}$, we see that

$$
\begin{aligned}
a \alpha^{i+2}\left(a^{\prime}\right) \in E\left(A^{\alpha^{i+2}}\right) & \Longleftrightarrow a a^{\prime} \in E(A) \\
& \Longleftrightarrow \alpha\left(\alpha^{-1}(a)\right) \alpha^{-1}\left(\alpha\left(a^{\prime}\right)\right) \in E(A) \\
& \Longleftrightarrow \alpha^{-1}(a) \alpha^{1}\left(a^{\prime}\right) \in E(A) \\
& \Longleftrightarrow \alpha^{-1}(a) \alpha^{i+1}\left(a^{\prime}\right) \in E\left(A^{\alpha^{i}}\right) \\
& \Longleftrightarrow \alpha^{-1}(a) \alpha^{-1}\left(\alpha^{i+2}\left(a^{\prime}\right)\right) \in E\left(A^{\alpha^{i}}\right)
\end{aligned}
$$

because $\alpha$ is an anti-automorphism of $A$. So $\alpha^{-1}: V\left(A^{\alpha^{i}}\right) \rightarrow V\left(A^{\alpha^{i+2}}\right)$ is also a homomorphism. Thus $\alpha$ is an isomorphism. So $A^{\alpha^{i}} \cong A^{\alpha^{i+2}}$.

COROLLARY 4.5. If $\alpha$ has odd order, then $A^{\alpha} \cong A$.

Proof. Let $\alpha$ be an anti-automorphism with odd order. By definition, there must be an odd number $p$ for which $\alpha^{p}=i d$. And thus $A^{\alpha^{p}}=A^{i d}=A$. Then by Lemma 4.4,

$$
A^{\alpha^{1}} \cong A^{\alpha^{3}} \cong A^{\alpha^{5}} \cong \cdots \cong A^{\alpha^{p}}
$$

since $p$ is odd. Thus we have $A^{\alpha} \cong A^{\alpha^{p}}=A$. 
COROLlARY 4.6. If $\alpha$ has order $2 p$ where $p$ is odd, then $A^{\alpha} \cong A^{\alpha^{p}}$ where $\alpha^{p}$ has order 2 . Proof. Because $p$ is odd, again by Lemma $4.4, A^{\alpha} \cong A^{\alpha^{p}}$. Then because $\alpha$ has order $2 p$, $\alpha^{2 p}=i d$. And thus $\left(\alpha^{p}\right)^{2}=i d$. So $\alpha^{p}$ has order 2 , which in turn gives us the fact that $\alpha^{p}$ is an anti-automorphism as well as an automorphism of order 2.

This final result does not require $\alpha$ to be an anti-automorphism. In chapter 3 , we found that for every $B$ satisfying $A \times C \cong B \times C$ there is a special permutation $\alpha$ such that $B \cong A^{\alpha}$. When enumerating all these digraphs $A^{\alpha}$, we are not interested in those that are isomorphic to one another. The following lemma proved in [5] helps divide these digraphs $A^{\alpha}$ into classes so that we can focus our attention on those digraphs $A^{\alpha}$ that are different.

LEMMA 4.7. For any digraph $A$ and permutations $\mu$ and $\lambda$ on the vertices of $A$, then $A^{\mu} \cong A^{\lambda}$ if and only if $\alpha^{-1} \mu \beta=\lambda$ for some permutations $\alpha$ and $\beta$ with $(\alpha)(\beta) \in E(A !)$.

Proof. First assume that $A^{\lambda} \cong A^{\mu}$ for permutations $\lambda$ and $\mu$ on the vertices of $A$. Then there exists an isomorphism $\psi: V\left(A^{\lambda}\right) \rightarrow V\left(A^{\mu}\right)$. Observe $\lambda=\left(\psi^{-1}\right) \mu\left(\mu^{-1} \psi \lambda\right)$. We will show $(\psi)\left(\mu^{-1} \psi \lambda\right) \in E(A !)$ to complete the proof. First observe $\psi$ and $\mu^{-1} \psi \lambda$ are permutations on the vertices of $A$, so they are in fact vertices in $A$ !. In order to show the edge exists in the factorial, consider any edge $a a^{\prime}$ in $A$. Then,

$$
\begin{aligned}
a a^{\prime} \in E(A) & \Longleftrightarrow a \lambda\left(a^{\prime}\right) \in E\left(A^{\lambda}\right) \\
& \Longleftrightarrow \psi(a) \psi \lambda\left(a^{\prime}\right) \in E\left(A^{\mu}\right) \\
& \Longleftrightarrow \psi(a) \mu^{-1} \psi \lambda\left(a^{\prime}\right) \in E(A) .
\end{aligned}
$$

So $(\psi)\left(\mu^{-1} \psi \lambda\right)$ is an edge in $A$ !.

Conversely, assume $\alpha^{-1} \mu \beta=\lambda$ for some permutations $\alpha$ and $\beta$ with $(\alpha)(\beta) \in E(A !)$. Recall $V(A)=V\left(A^{\lambda}\right)=V\left(A^{\mu}\right)$. So consider the mapping $\alpha: V\left(A^{\lambda}\right) \rightarrow V\left(A^{\mu}\right)$. This 
is a bijection because $\alpha$ is a permutation on $V(A)$. Once we show that $\alpha$ and $\alpha^{-1}$ are homomorphisms, we will have an isomorphism between $A^{\lambda}$ and $A^{\mu}$. Consider the following:

$$
\begin{aligned}
a a^{\prime} \in E\left(A^{\lambda}\right) & \Longleftrightarrow a \lambda^{-1}\left(a^{\prime}\right) \in E(A) \\
& \Longleftrightarrow \alpha(a) \beta \lambda^{-1}\left(a^{\prime}\right) \in E(A) \\
& \Longleftrightarrow \alpha(a) \mu \beta \lambda^{-1}\left(a^{\prime}\right) \in E\left(A^{\mu}\right) \\
& \Longleftrightarrow \alpha(a) \alpha \alpha^{-1} \mu \beta \lambda^{-1}\left(a^{\prime}\right) \in E\left(A^{\mu}\right) \\
& \Longleftrightarrow \alpha(a)(\alpha)\left(\alpha^{-1} \mu \beta\right)\left(\lambda^{-1}\right)\left(a^{\prime}\right) \in E\left(A^{\mu}\right) \\
& \Longleftrightarrow \alpha(a)(\alpha)(\lambda)\left(\lambda^{-1}\right)\left(a^{\prime}\right) \in E\left(A^{\mu}\right) \\
& \Longleftrightarrow \alpha(a) \alpha\left(a^{\prime}\right) \in E\left(A^{\mu}\right)
\end{aligned}
$$

So $\alpha$ and $\alpha^{-1}$ are homomorphisms. Thus $\alpha$ is an isomorphism, giving us $A^{\lambda} \cong A^{\mu}$. 
Bibliography 


\section{Bibliography}

[1] L. Culp, An Isomorphism Theorem for Digraphs, Masters Thesis Virginia Commonwealth University (2009).

[2] R. Hammack, On direct product cancellation of graphs, Discrete Mathematics, 309 (2009) 2538-2543.

[3] P. Hell and J. Nešetřil, Graphs and Homomorphisms, Oxford Lecture Series in Mathematics, Oxford U. Press, (2004).

[4] L. Lovász, On the cancellation law among finite relational structures, Periodica Mathematica Hungarica 1(2) (1971) 145-156.

[5] K. Toman, Cancellation of Direct Products of Digraphs, Masters Thesis Virginia Commonwealth University (2009). 
Vita

Heather was born in Tallahassee, Florida and moved to Virginia at the age of four. She was homeschooled through middle school and high school. During the eleventh grade, she starting taking college classes, completing 33 credit hours by the end of her senior year. She attended Houghton College in Western New York where she majored in Mathematics and Adolescence Education. In 2008, Heather graduated summa cum laude from Houghton. The following fall, Heather began work on her masters degree in pure mathematics at Virginia Commonwealth University. While at VCU, she taught College Algebra and Precalculus. This confirmed her desire to teach college level math as a career. During her time at VCU she was introduced to Graph Theory and will be pursuing a doctorate in this field at the University of South Carolina beginning in the fall of 2010.

When Heather is not studying or working on lesson plans, she enjoys spending time with her parents and younger brother at home, during their weekly night out, and on vacations. She also loves spending time outdoors with her Labrador Retrievers which her family breeds. Weekends are filled with church activities at Remlik Wesleyan Church where Heather's father is the senior pastor. And in those few spare moments in the evenings, she will pick up her crocheting which she learned from her grandmother. In addition to pure math, Heather has learned much about accounting, working with her father and at a non-profit organization over the summer. 